\title{
Complete sequences of organelle genomes from the medicinal plant Rhazya stricta (Apocynaceae) and contrasting patterns of mitochondrial genome evolution across asterids
}

\author{
Seongjun Park', Tracey A Ruhlman'1, Jamal SM Sabir², Mohammed HZ Mutwakil², Mohammed N Baeshen²,
} Meshaal J Sabir ${ }^{2}$, Nabih A Baeshen ${ }^{2}$ and Robert K Jansen ${ }^{1,2^{*}}$

\begin{abstract}
Background: Rhazya stricta is native to arid regions in South Asia and the Middle East and is used extensively in folk medicine to treat a wide range of diseases. In addition to generating genomic resources for this medicinally important plant, analyses of the complete plastid and mitochondrial genomes and a nuclear transcriptome from Rhazya provide insights into inter-compartmental transfers between genomes and the patterns of evolution among eight asterid mitochondrial genomes.

Results: The 154,841 bp plastid genome is highly conserved with gene content and order identical to the ancestral organization of angiosperms. The 548,608 bp mitochondrial genome exhibits a number of phenomena including the presence of recombinogenic repeats that generate a multipartite organization, transferred DNA from the plastid and nuclear genomes, and bidirectional DNA transfers between the mitochondrion and the nucleus. The mitochondrial genes sdh3 and rps 14 have been transferred to the nucleus and have acquired targeting presequences. In the case of rps 14, two copies are present in the nucleus; only one has a mitochondrial targeting presequence and may be functional. Phylogenetic analyses of both nuclear and mitochondrial copies of rps 14 across angiosperms suggests Rhazya has experienced a single transfer of this gene to the nucleus, followed by a duplication event. Furthermore, the phylogenetic distribution of gene losses and the high level of sequence divergence in targeting presequences suggest multiple, independent transfers of both sdh 3 and rps 14 across asterids. Comparative analyses of mitochondrial genomes of eight sequenced asterids indicates a complicated evolutionary history in this large angiosperm clade with considerable diversity in genome organization and size, repeat, gene and intron content, and amount of foreign DNA from the plastid and nuclear genomes.

Conclusions: Organelle genomes of Rhazya stricta provide valuable information for improving the understanding of mitochondrial genome evolution among angiosperms. The genomic data have enabled a rigorous examination of the gene transfer events. Rhazya is unique among the eight sequenced asterids in the types of events that have shaped the evolution of its mitochondrial genome. Furthermore, the organelle genomes of $R$. stricta provide valuable genomic resources for utilizing this important medicinal plant in biotechnology applications.
\end{abstract}

Keywords: Asterids, Gene transfers, Medicinal plant, Organelle genomes, Harmal, Adfir

\footnotetext{
* Correspondence: jansen@austin.utexas.edu

'Department of Integrative Biology, University of Texas at Austin, Austin, TX 78712, USA

2Department of Biological Science, Faculty of Science, King Abdulaziz University, Jeddah 21589, Saudi Arabia
}

\section{Biomed Central}

(c) 2014 Park et al.; licensee BioMed Central Ltd. This is an Open Access article distributed under the terms of the Creative Commons Attribution License (http://creativecommons.org/licenses/by/4.0), which permits unrestricted use, distribution, and reproduction in any medium, provided the original work is properly credited. The Creative Commons Public Domain Dedication waiver (http://creativecommons.org/publicdomain/zero/1.0/) applies to the data made available in this article unless otherwise stated. 


\section{Background}

Rhazya stricta (Apocynaceae) is among the most economically important medicinal plants adapted to the arid regions of South Asia and the Middle East. Leaf extracts are prescribed in folk medicine for the treatment of various disorders including diabetes, sore throat, syphilis, helminthiasis, inflammatory conditions and rheumatism [1-3]. At least 100 alkaloids have been isolated and identified from this species [4,5]. A recent study by Baeshen et al. [6] found that $R$. stricta extracts promoted apoptosis induction in breast cancer cells, suggesting its potential as a chemopreventive or therapeutic agent. However, currently there are no genomic resources for $R$. stricta to facilitate the development of this species for therapeutic applications using a natural products genomics approach $[7,8]$.

The availability of plant genomic data has improved dramatically in recent years through the development of next-generation sequencing (NGS) technologies [9-11] and improved assembly methods [12]. Genomic resources that provide information about gene content and the metabolic pathways that produce compounds with pharmaceutical value are paramount to the potential for improvement and application of natural products as therapeutics.

Most plastid genomes have a quadripartite structure with an inverted repeat separated by large and small single-copy regions, an arrangement that is highly conserved across land plants [13]. Plastid genome sizes of photosynthetic land plants range from 107 to $217 \mathrm{~kb}$ and contain 101-118 different genes with majority of these coding for proteins involved in photosynthesis and gene expression along with transfer RNA (tRNA) and ribosomal RNA (rRNA) genes [14].

In contrast mitochondrial genomes are remarkably variable among land plants. These genomes are usually larger than plastid genomes, ranging from $105 \mathrm{~kb}$ in the moss Physcomitrella patens [15] to $11.3 \mathrm{Mb}$ in the angiosperm Silene conica [16]. Despite their relatively large size, mitochondrial genomes contain fewer genes than their plastid counterparts; 37-83 different genes including protein coding, tRNA and rRNA genes [17]. Plant mitochondrial genomes map as circular or noncircular structures and have a dynamic, multipartite organization due to active recombination associated with repeat regions that can be as large as $109 \mathrm{~kb}$ in Tripsacum dactyloides [18-22]. Rearrangements in plant mitochondrial genomes are facilitated by homologs of bacterial DNA repair proteins such as $\operatorname{RecA}$ and MutS [23] and often result in chimeric open reading frames (ORFs) [22,24]. One of the most prominent characteristics of plant mitochondrial genomes is the presence of foreign DNA including DNA that has been transferred from the plastid, nucleus and even genetic material from other species [25-29]. RNA editing is common in plant mitochondrial genomes and usually involves the conversion of cytidine (C) to uracil (U) [30,31].
Among angiosperms there is a high frequency of organellar DNA transferred to the nucleus. Although most functional gene transfers from organelles to the nucleus occurred shortly after their endosymbiotic origin, gene transfer is an ongoing process [25]. While transfer of organellar DNA to the nucleus is rampant, acquisition of function is much less common. Transferred organelle genes must acquire nuclear expression elements and target peptides to shuttle the gene product back to the organelle (referred to as a transit peptide in the case of plastids or a presequence for mitochondria) [32]. Transferred organelle genes may acquire a novel targeting sequence or adopt one from an existing nuclear gene [33-35]. In a few cases, mitochondrial genes have been transferred without the acquisition of $\mathrm{N}$ terminal sequences. In these cases, gene products may be targeted to the mitochondrion by internal features of the polypeptide [36].

This report provides the complete sequences of the plastid and mitochondrial genomes of $R$. stricta, a medicinally important species. Genome organization is characterized including identification of the gene transfers between the mitochondria and the nucleus. In addition, the mitochondrial genome organization of $R$. stricta is compared to seven previously published asterid genomes to examine patterns of organelle genome evolution across this large angiosperm clade.

\section{Results}

\section{Plastid genome organization of Rhazya stricta}

The $R$. stricta plastid genome is 154,841 bp in length with a pair of inverted repeats (IRa and IRb) of 25,513 bp separated by small and large single-copy (SSC and LSC) regions of 17,745 and 86,070 bp, respectively (Figure 1 and Table 1). The GC content is $37.6 \%$, and the genome consists of $50.7 \%$ protein-coding genes, $41.6 \%$ non-coding regions, $1.8 \%$ tRNA and $5.8 \%$ rRNA genes. The Rhazya plastid genome encodes 114 genes, 16 of which are duplicated in the IR, for a total of 130 genes. There are 80 protein-coding genes, 30 tRNA and 4 rRNA genes (Table 1). The IR has expanded slightly at both the IR/LSC and IR/SSC boundaries relative to $N$. tabacum. The expansion encompassed the first 91 nucleotides of rps19 in IRb, generating an rps19 fragment in IRa, and extended into the SSC to include the stop codon of the $n d h F$ gene. Mitochondrial-like gene sequences were not detected in the Rhazya plastid genome.

\section{Mitochondrial genome organization of Rhazya stricta Genome size and content}

The $R$. stricta mitochondrial genome assembled into a single master chromosome of 548,608 bp (Figure 1). The GC content is $43.7 \%$, and the genome consists of $6.7 \%$ protein-coding genes, $92.1 \%$ intergenic spacers, $0.2 \%$ tRNA and $1.0 \%$ rRNA genes (Table 1). The genome 


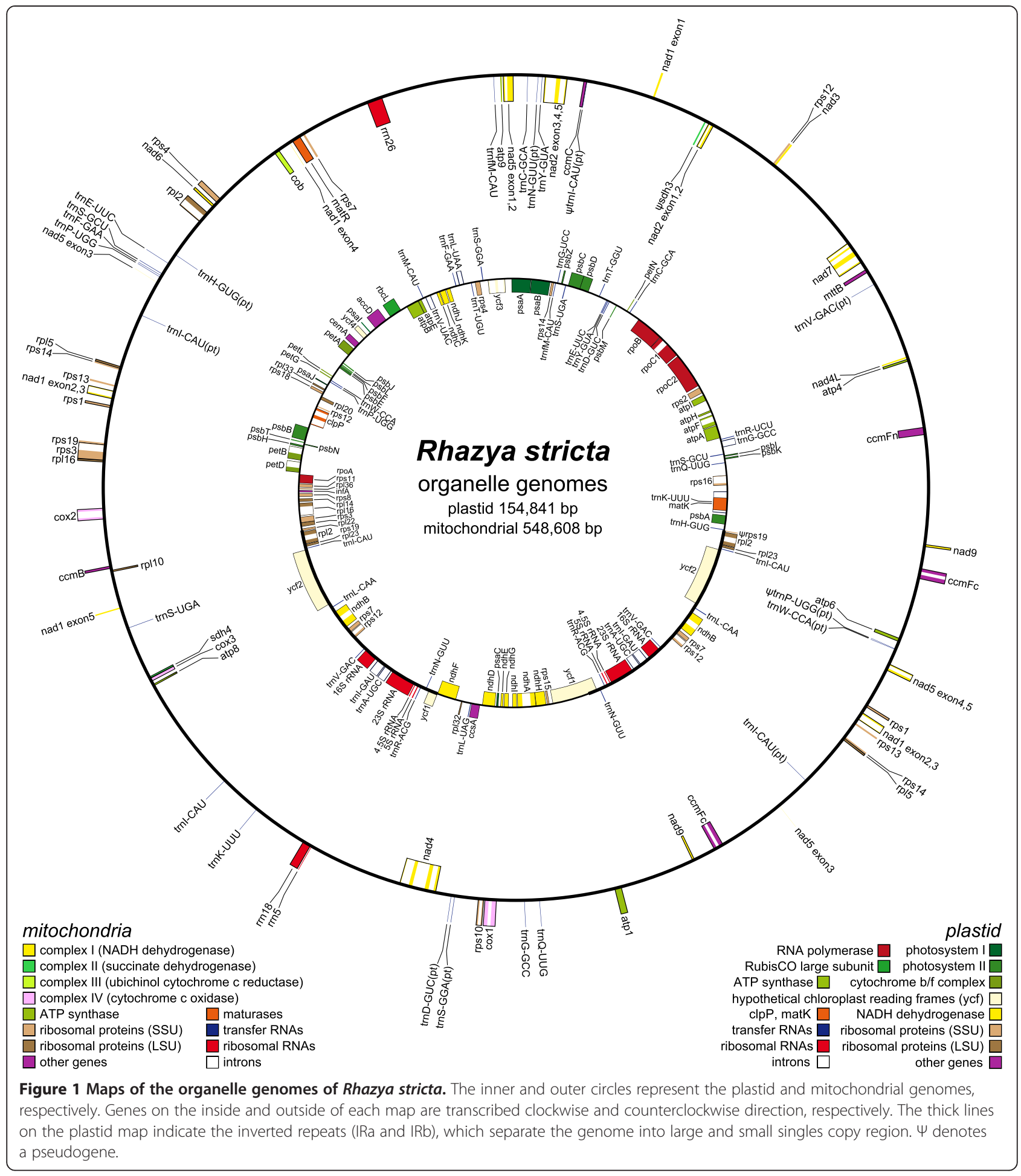

contains 53 genes including 38 protein-coding genes, 12 tRNAs, and 3 rRNAs (Table 1; Additional file 1: Table S1). Two identical copies of the genes nad9, ccmFc, rpl15, rps1, rps13 and rps14 were identified in two large repeats (Additional file 1: Table S1). The ribosomal protein genes rps 2 and rps11 are absent and respiratory protein gene sdh3 appeared to be a pseudogene as it lacks a start codon. The rps 14 gene has an 8 bp deletion near the 3' end resulting in a frame shift that truncates the sequence relative to other angiosperms. The $\mathrm{N}$-terminal portion of the atp6 gene is truncated, having lost 125 amino acids relative to the atp 6 gene of Nicotiana tabacum. The 
Table 1 Characteristics of Rhazya stricta organelle genomes

\begin{tabular}{lcc}
\hline Genome characteristic & Plastid genome & Mitochondrial genome \\
\hline Genome size (bp) & 154,841 & 548,608 \\
GC content (\%) & 37.6 & 43.7 \\
Genes & & \\
Protein coding genes & $80(5)$ & $38(6)$ \\
$\quad(\%)$ & 50.7 & 6.7 \\
tRNA genes & $30(7)$ & 12 \\
$\quad(\%)$ & 1.8 & 0.2 \\
rRNA genes & $4(4)$ & 3 \\
$\quad(\%)$ & 5.8 & 1.0 \\
Introns & & 18 \\
cis-spliced group & $20(5)$ & 6 \\
trans-spliced group & 1 & $4(2)$ \\
Plastid-derived & & $7(1)$ \\
Protein-coding genes & & \\
tRNA genes & &
\end{tabular}

The numbers in parentheses indicate the number of genes duplicated in repeat region.

Rhazya atp6 has only a small portion of the gene containing the conserved ATP synthase F0 subunit 6 domain (pfam00119). The cox1 group I intron was identified as well as 23 group II introns, six of which require transsplicing (Table 1).

\section{Repeat structure}

BlastN analysis of the Rhazya mitochondrial genome against itself revealed 77,887 bp of repetitive DNA, ranging from 39 to $36,624 \mathrm{bp}$ in length (Additional file 1: Table S2). The repetitive DNA constitutes $14.2 \%$ of the genome including $12.9 \%$ large ( $>1 \mathrm{~kb}$ ), $0.6 \%$ intermediate $(100-1000 \mathrm{bp})$ and $0.7 \%$ small $(<100 \mathrm{bp})$ repeats. Mapping of corrected PacBio reads against the mitochondrial genome revealed conflicts between the PacBio reads and the assembled genome suggesting the existence of subgenomic circles (Additional file 2: Figure S1A); the long PacBio reads spanned the junctions of the repeat regions in the assembled master chromosome. The conflicts were associated with two large repeats $(36,251$ and 32,072 bp) and five intermediate-sized repeats (559, 508, 281, 252, and 124 bp) (Additional file 2: Figure S1B). Most of the repeats clustered together sequentially in twos, threes or fives. PCR confirmed the existence of isomeric and/or subgenomic circles that arose as a result of repeat-mediated homologous recombination among the seven repeat families (Additional file 2: Figure S2).

\section{Transposable elements}

The Rhazya mitochondrial genome contains 16,008 bp (2.9\%) of transposable elements (TEs) of many different types (Additional file 1: Table S3) the majority of which are copia- and gypsy-like retrotransposons (12,604 bp). Most TEs were identified in intergenic regions (Figure 2) with only 1,095 bp (6.8\%) inserted into genic regions (Additional file 1: Table S4).

\section{Open reading frames}

One hundred twenty-three mitochondrial ORFs ( $\geq 300 \mathrm{bp}$ in length) were identified in Rhazya intergenic regions (Additional file 1: Table S5). Blast searches against the non-redundant nucleotide and protein databases in the NCBI identified several ORFs with high similarity to hypothetical proteins, some of which were cytoplasmic male sterile (CMS) related ORFs, or were derived from intergenic regions of other plant mitochondrial genomes. Other ORFs contained sequences similar to organellar or viral DNA and RNA polymerases, retrotransposons or nuclear genes. The remaining ORFs had no significant similarity to any genes or proteins in the NCBI database.

BlastN queries of the Rhazya transcriptome assembly (subject database) detected transcripts for many of the 123 ORFs (Additional file 1: Table S5). One transcript had a sequence identical to a mitochondrial copy of a nuclear gene, (R)-mandelonitrile lyase, which is present as split ORFs in the mitochondrial genome of Rhazya due to a 5 bp deletion relative to the nuclear gene (Additional file 2: Figure S3). RT-PCR confirmed that the transcript has $100 \%$ sequence identity to the mitochondrial DNA sequence, including the nonsense mutation.

Twelve ORFs of at least $150 \mathrm{bp}$ in length, three of which are present as two copies, appeared to be chimeric ORFs that contain small fragments ( $>30 \mathrm{bp}$ ) of mitochondrial genes (Additional file 1: Table S6). Five of these ORFs were predicted to encode one or two transmembrane helices. ORF56b contains small fragments of the three mitochondrial genes, $r p l 2$, matR and $c c m F n$, that overlap with repeat 6 (Additional file 2: Figure S4A). Two ORFs are located in the region with three or four repeats that are associated with atp6 (Additional file 2: Figure S4B). While each of these two were predicted to encode a protein with two transmembrane helices, neither could be identified by a BlastN search against annotated Rhazya mitochondrial genes.

\section{Sequences of plastid origin}

Plastid-like sequences were found in 38 fragments throughout the Rhazya mitochondrial genome, ranging from 75 to 5,069 bp in length (Figure 2; Additional file 1: Table S7). The total amount of plastid sequence was $32,810 \mathrm{bp}$, representing $6.0 \%$ of the mitochondrial genome. The 38 insertion regions represent $24.1 \%$ of the Rhazya plastid genome. Six intact plastid genes, $n d h H$, $a t p H, p s a B(x 2), p s a A(x 2)$, eight tRNAs (one of which had two copies), six pseudogenes and numerous partial 


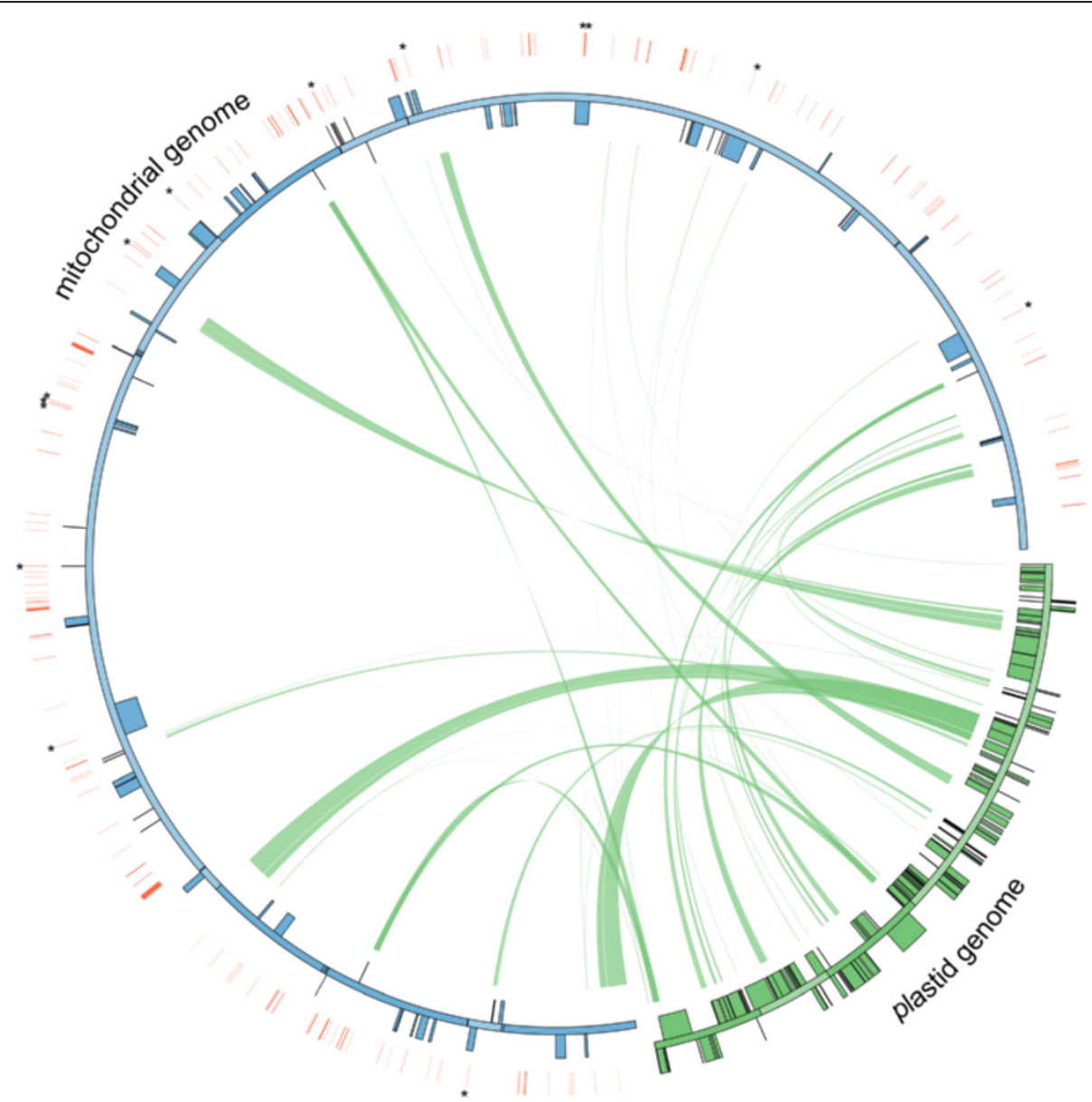

Figure 2 Schematic representation of transfers of plastid DNA and transposable elements into the mitochondrial genome of Rhazya stricta. Each green line within the circle shows the regions of the plastid genome that have been inserted into different locations of the mitochondrial genome. Red lines outside of the mitochondrial genome indicate the location of integrated transposable elements (TEs) and asterisks indicate TEs that have inserted into genic regions. Genes indicated as blue and green boxes on the inside and outside of maps are transcribed clockwise and counterclockwise direction, respectively.

genes and intergenic spacer regions were identified. All plastid fragments were located in intergenic regions. Among the tRNAs, four differed by one to three nucleotide substitutions from copies in the plastid (one trnD-GUC, two trnH-GUG, two trnS-GGA, and three trnN-GUU). There are two degenerate copies of trnI-CAU and trnPUGG with six indels compared to the plastid-encoded tRNAs. Plastid-derived trnM-CAU was not detected in the Rhazya mitochondrial genome.

\section{Mitochondrial RNA editing}

PREP-Mt and PREPACT predicted 462 and 480 putative C-to-U RNA editing sites, respectively, in the 38 Rhayza mitochondrial protein-coding genes (Additional file 1: Table S8). Cytochrome c proteins and NADH dehydrogenase subunits (complex I) were more highly edited than other protein-coding genes, whereas ribosomal proteins had fewer edited sites. Available transcriptome data for 11 genes (atp1, atp9, cox1, cox2, cox3, nad4, nad5, nad7, rpl5, rps4, rps7) confirmed that of the 157 sites predicted by PREP-Mt for these genes, 148 (96\%) sites were edited (Additional file 1: Table S9). In the Rhazya mitochondrial genome, atp6 and rps10 begin with an ACG start codon instead of the standard ATG. In the case of rps10, PREP-MT predicted that the start codon was altered by RNA editing (score 1.0) to the standard ATG codon, whereas the editing prediction for atp 6 was only 0.25 . RNA editing was also predicted to generate the stop codons in atp6, atp9, and rps10 (score 1.0).

\section{Characterization of putative functional gene transfers to the nucleus}

All 38 protein-coding genes in the Rhazya mitochondrial genome were used to query the Rhazya transcriptome assembly (133,266 contigs). An ORF with $77.7 \%$ nucleotide sequence identity to the rps 14 gene was identified that includes a 5' extension of 162 bp (Figure 3A and 3B). The first 51 amino acids of this ORF were predicted 

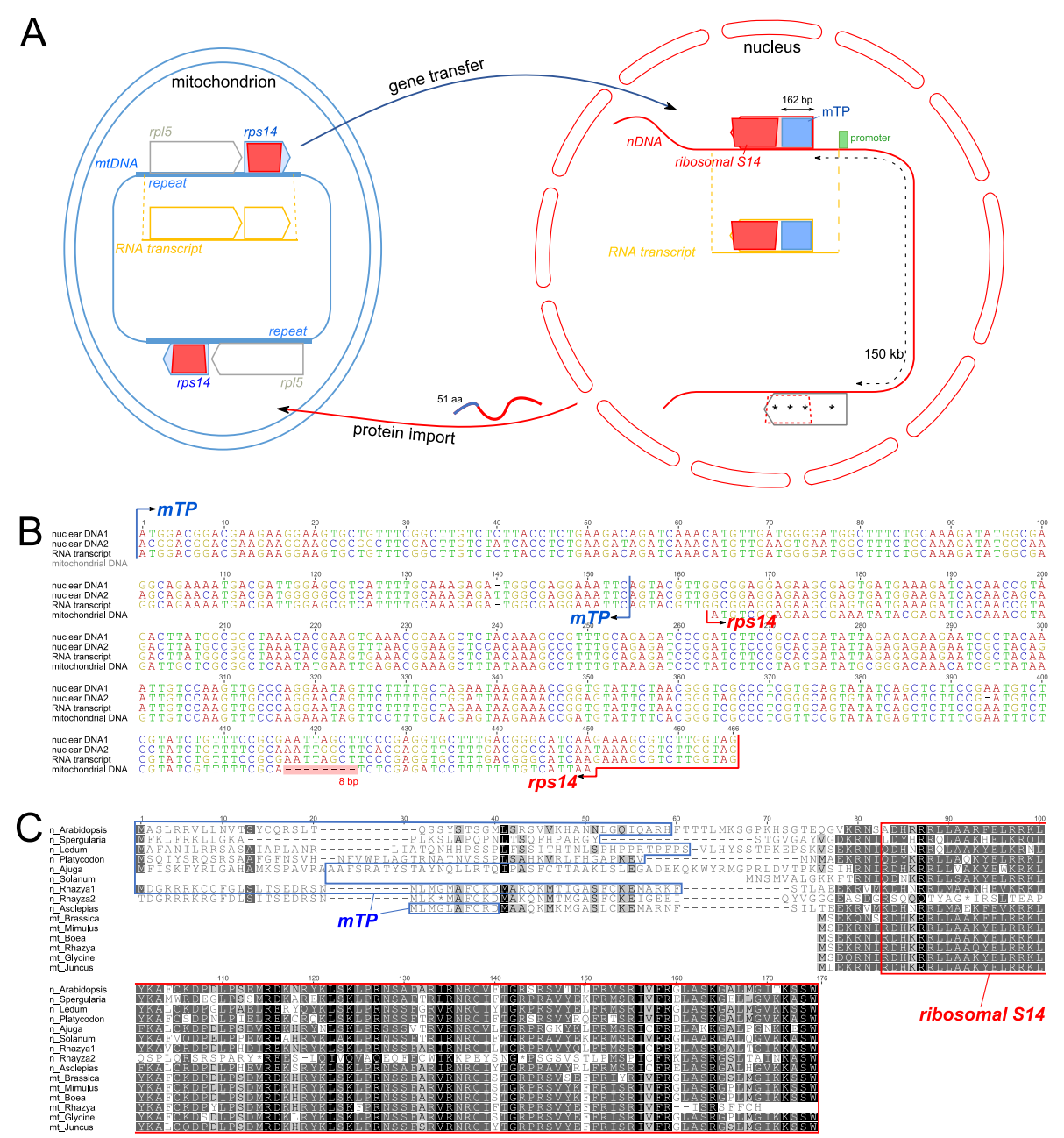

Figure 3 Gene transfer of $r$ s 14 . A. Schematic diagram of rps 14 gene transfer from mitochondrial genome to the nucleus. The mitochondrial rps 14 copies are identified in two repeat regions and are co-transcribed with rp/5. Boxes indicate mitochondrial targeting presequence (mTP; blue) and a conserved domain (ribosomal S14; red). The grey box arrow in the nuclear genome represents the non-functional copy of rps 14 and the dotted red line indicates a conserved domain. Internal stop codons are indicated with asterisks. B. Nucleotide sequence alignment of the two nuclear, transcript, and mitochondrial copies of rps 14 from Rhazya stricta. Shaded red box shows an 8 bp deletion that caused a frameshift. C. Amino acid sequence alignment of two nuclear and one mitochondrial copy of rps 14 of Rhazya with seven nuclear-encoded and five mitochondrial copies from other angiosperms (see Additional file 1: Table S13). Blue boxes indicate mitochondrial targeting presequences. Red boxes indicate the conserved domain of ribosomal S14. $\mathrm{mt}=$ mitochondrial, $\mathrm{n}=$ nuclear.

by TargetP to be an $\mathrm{mTP}$ (mitochondrial $=0.6$ ), whereas Predotar returned the prediction of 'elsewhere' (elsewhere $=1.0)$. Examination of a draft $R$. stricta de novo nuclear genome sequence (D. Arasappan, unpublished) confirmed the presence of an intronless, nuclearencoded rps14 gene showing 100\% nucleotide sequence identity to the transcript (Figure 3B). Another copy of rps 14 with $90.6 \%$ nucleotide sequence identity was detected on the same scaffold approximately $150 \mathrm{~kb}$ from the first copy, however it lacks a proximal start codon and has four internal stop codons (Figure 3A and $3 \mathrm{C}$ ). Phylogenetic analysis of nuclear and mitochondrial copies of rps14 showed that the Rhazya mitochondrial copy did not group with other asterids but instead was positioned sister to the clade that includes nuclear copies from other angiosperms (Figure 4A). However, support for this placement was very weak $(<50 \%$ bootstrap value) and resolution of relationships among the mitochondrial copies was low. The two nuclear copies of $R$. stricta grouped together with high bootstrap support (94\%), and branch lengths on the tree indicate that the putative non-functional copy (i.e. the one lacking an mTP) has experienced much higher substitution rates (Rhazya stricta 2 in Figure 4A). Protein sequence alignment of the extended amino acids upstream from rps14 was highly divergent with very low identities between Rhazya 


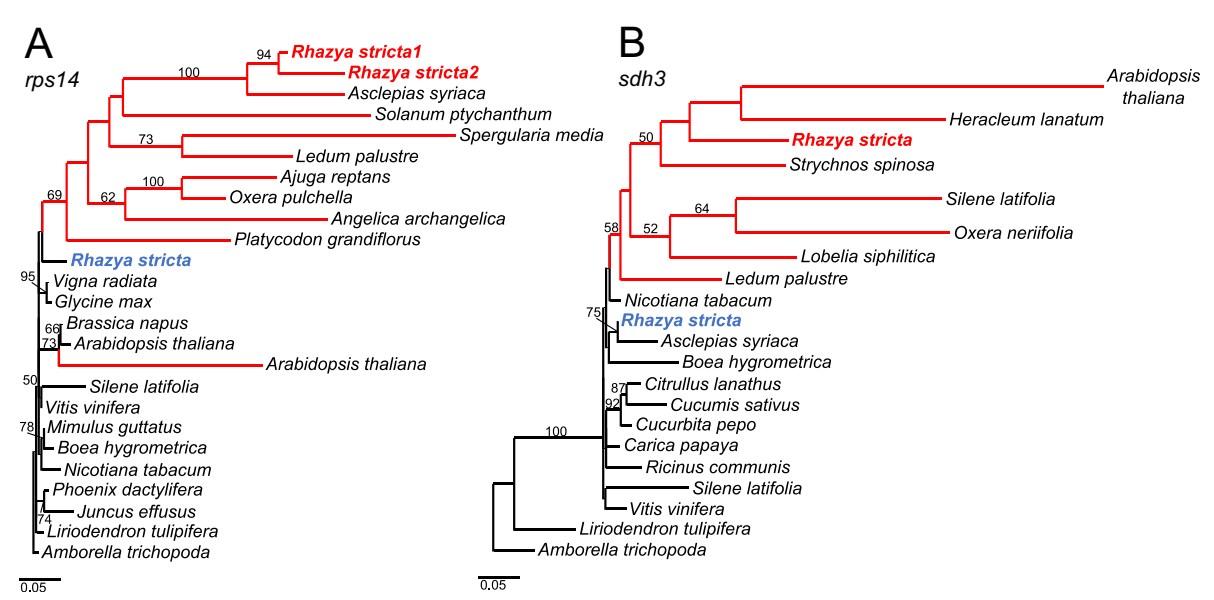

Figure 4 Maximum likelihood phylogenetic trees for mitochondrial genes of Rhazya transferred to the nucleus. A. Mitochondrial and nuclear rps 14 sequences of angiosperms. B. Mitochondrial and nuclear sdh3 sequences of angiosperms. Bold font indicates mitochondrial (blue) and nuclear copies (red) of rps 14 and sdh3 in Rhazya stricta. Bootstrap support values $>50 \%$ are shown at nodes. Red and black lines indicate nuclear and mitochondrial sequences, respectively.

and seven other species, ranging from $12.5 \%$ to $75.8 \%$ (Figure 3C). Protein sequence alignment of the nuclear copy of Rhazya rps14 to mitochondrial-encoded sequences from five species of seed plants showed pairwise identities ranging from $73.1 \%$ to $75.3 \%$, higher than the pairwise identity between the nuclear and mitochondrial Rhazya rps 14 copies at $65.5 \%$ (Figure 3C).

The Rhazya transcriptome assembly was queried with the $s d h 3$ pseudogene sequence from the mitochondrial genome. A contig containing an sdh3-like ORF with $81.5 \%$ nucleotide sequence identity to the query sequence was detected (Figure 5A and 5B). The ORF included a 5 ' extension of $555 \mathrm{bp}$ and the first 66 amino acids of the ORF were predicted by TargetP (0.8) and Predotar (0.9) to be an mTP. Phytozome predicted a small heat shock protein ( $h s p 22$ ) between the targeting presequence and the sdh3 coding region (Figure 5B and 5C). Examination of the draft $R$. stricta de novo nuclear genome assembly confirmed the presence of the nuclear-encoded sdh3 gene containing two exons totaling 960 bp separated by a $105 \mathrm{bp}$ intron (Figure 5A and 5B). Phylogenetic analysis of nuclear and mitochondrial copies of $s d h 3$ showed that the Rhazya mitochondrial copy was nested within a clade of asterid mitochondrial copies and the nuclear-encoded copy grouped within a nuclear-encoded clade (Figure 4B). Protein sequence alignment of the predicted targeting presequence and the remaining sequence upstream from sdh3 was highly divergent with low amino acid identities between Rhazya and five other species, ranging from $12.2 \%$ to $22.2 \%$ (Figure 5C). Protein sequence alignment of the nuclear copy of Rhazya sdh3 to nuclear- and mitochondrial-encoded sequences from several species revealed low amino acid identities of 50.0-59.4\% and 46.4$54.9 \%$, respectively (Figure 5 C).

\section{Comparison of Rhazya mitochondrial genome to seven other asterids}

Mitochondrial genome sizes of the eight asterids range from 281,132 bp in Daucus to 682,498 bp in Asclepias (Figure 6A; Additional file 1: Table S10). Using BlastN searches (e-value 1e-6), Rhazya was found to share more sequences $(214 \mathrm{~kb})$ with Asclepias, both coding and noncoding, than with other asterids (Nicotiana - $171 \mathrm{~kb}$, Mimulus - 168 kb, Boea - 146 kb, Daucus - 148 kb, Helianthus - $125 \mathrm{~kb}$, and Vaccinium - $128 \mathrm{~kb}$ ). GC content across the eight genomes ranges from $43.3 \%$ in Boea to 45.4\% in Daucus (Additional file 1: Table S10). Only one colinear gene block was identified among the eight asterid genomes: rrn18-rrn5 (Additional file 2: Figure S5).

BlastN searches of each mitochondrial genome against its plastid counterpart revealed that plastid derived sequences account for $2.5-6.9 \%$ of the eight asterid mitochondrial genomes (Figure 6A; Additional file 1: Table $\mathrm{S} 10)$. The number of tRNAs derived from plastids varies among the eight asterids, ranging from five in Daucus to 11 in Boea (Additional file 1: Table S11). All asterids examined share four (trnD-GUC, trnH-GUG, trnN-GUU, and $\operatorname{trn}(\mathrm{W}-\mathrm{CC} A)$ intact plastid-derived tRNAs. In addition to plastid-derived sequences, the eight asterid mitochondrial genomes contain 2.9-6.0\% TEs (Figure 6A; Additional file 1: Table S10), most of which were LTRretrotransposons (Figure 6B). The percentage of TEs in Rhazya is the lowest among the asterids examined.

Repetitive DNA content among asterid mitochondrial genomes is highly variable, ranging from $1.0 \%$ in Boea to 19.4\% in Daucus (Figure 6A; Additional file 1: Table S10). All asterids had numerous small repetitive DNAs ( $<100$ bp), with the greatest number found in Vaccinium (Figure 6C; Additional file 1: Table S10). The mitochondrial genome of 


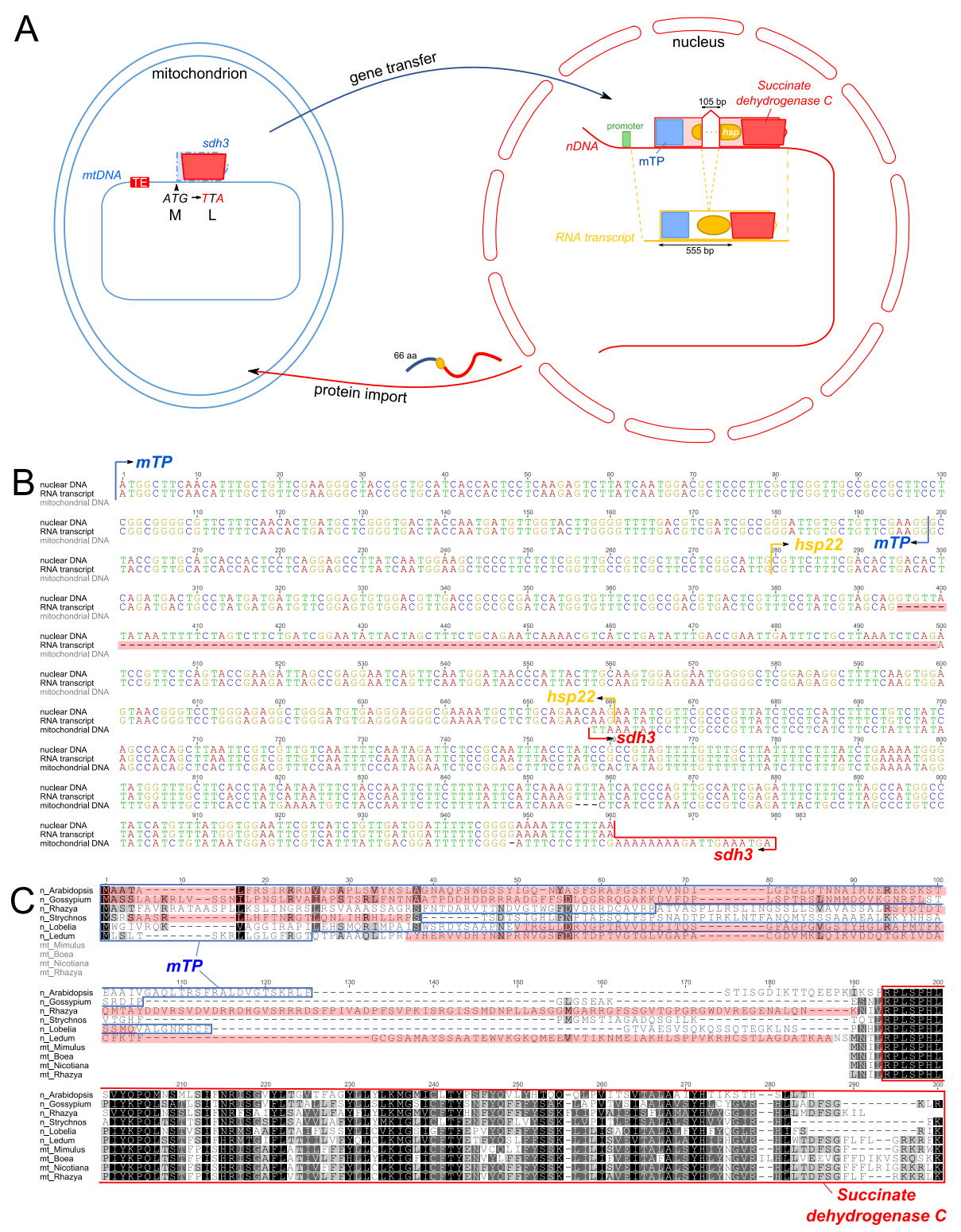

Figure 5 Gene transfer of $s d h 3$. A. Schematic diagram of $s d h 3$ gene transfer from mitochondrial genome to the nucleus. A transposable element (TE) insertion is shown in red in the mitochondrial DNA. The dashed line outlines a pseudogene due to mutations in the start codon as indicated. Boxes and oval indicate mitochondrial targeting presequence (mTP; blue), heat shock protein (hsp; orange), and a conserved domain (succinate dehydrogenase $C_{;}$red). B. Nucleotide sequence alignment of the nuclear, transcript, and mitochondrial copies of Rhazya stricta. The shaded red area shows the intron within predicted heat shock protein (hsp22). C. Amino acid sequence alignment of Rhazya nuclear and mitochondrial sdh3 with six nuclear-encoded and three mitochondrial-encoded copies from other angiosperms (see Additional file 1: Table S13). Boxes indicate mitochondrial targeting presequence (blue) and a conserved domain of succinate dehydrogenase $C$ (red), and shaded red boxes indicate the remaining portions of genes into which sdh3 was transferred (heat shock proteins, Rhazya and Gossypium-hsp22, Arabidopsis-hsp70; Iron-sulfur cluster scaffold-like protein, Ledum; hypervariable Bacillus group-specific protein, Strychnos; Pyrimidine (PYR) binding domain of thiamine pyrophosphate (TPP)-dependent enzyme, Lobelia). $\mathrm{mt}=$ mitochondrial, $\mathrm{n}=$ nuclear.

Rhazya contains longer repeats, whereas Daucus had a higher percentage of repetitive DNA. Repetitive DNA is widely scattered across the eight asterid genomes, and each genome contains a unique pattern of repeats (Figure 7).

The phylogenetic distribution of genes and introns among the eight asterids and one representative species each from Caryophyllales and rosids is shown in Figure 8 . This analysis revealed a number of shared and unique gene and intron gains/losses among asterids. The asterid mitochondrial genomes share 31 proteincoding, 3 rRNA and 12 tRNAs, however gene and intron content across asterids varies considerably for the 

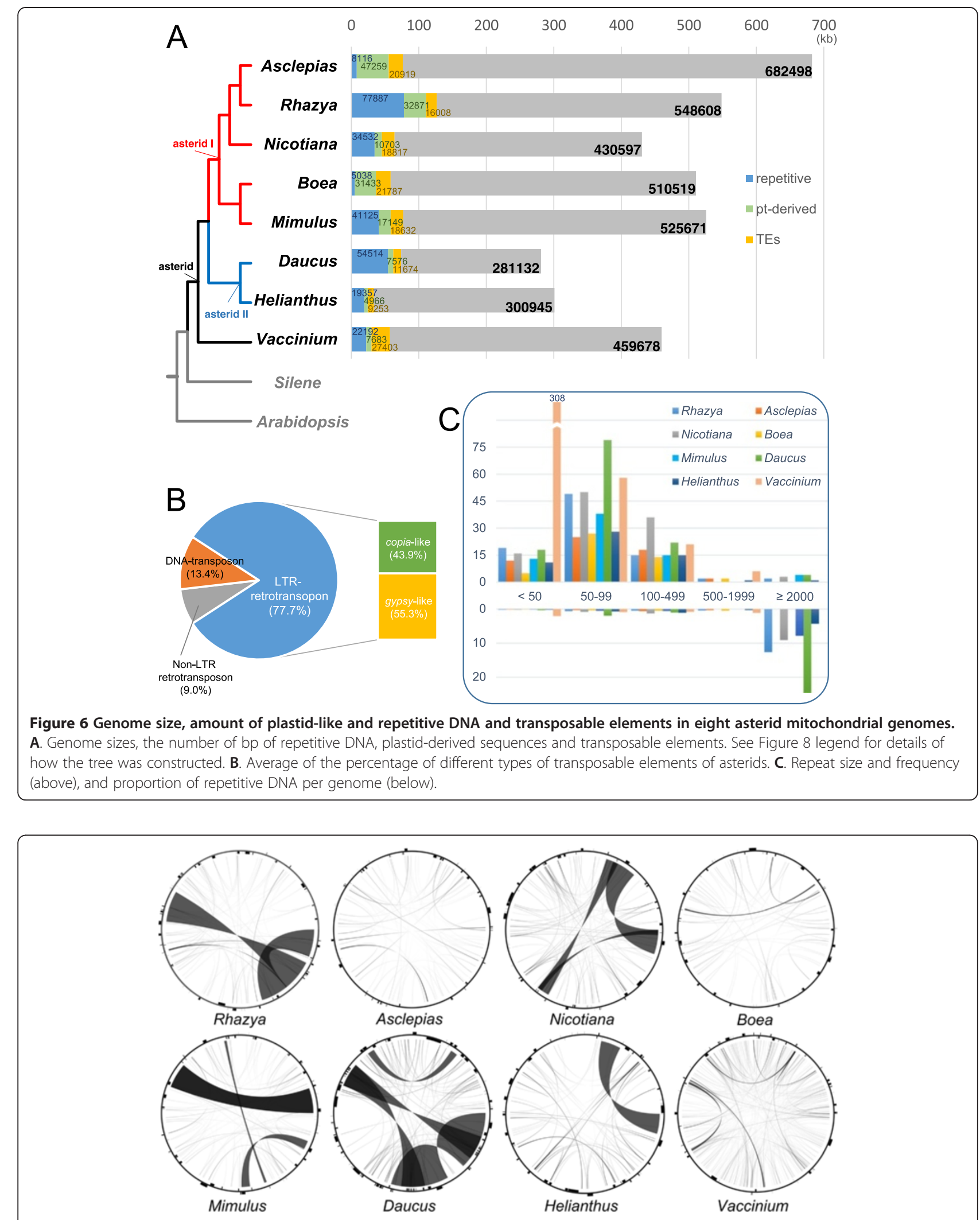

Figure 7 Distribution of repetitive DNA in Rhazya mitochondrial genome compared to seven other asterids. Black lines within circular maps indicate the positions of the pairs of repeats, with crossed connecting lines denoting reverse repeats. Black boxes on the inner and outer circle indicate the positions of mitochondrial genes. 


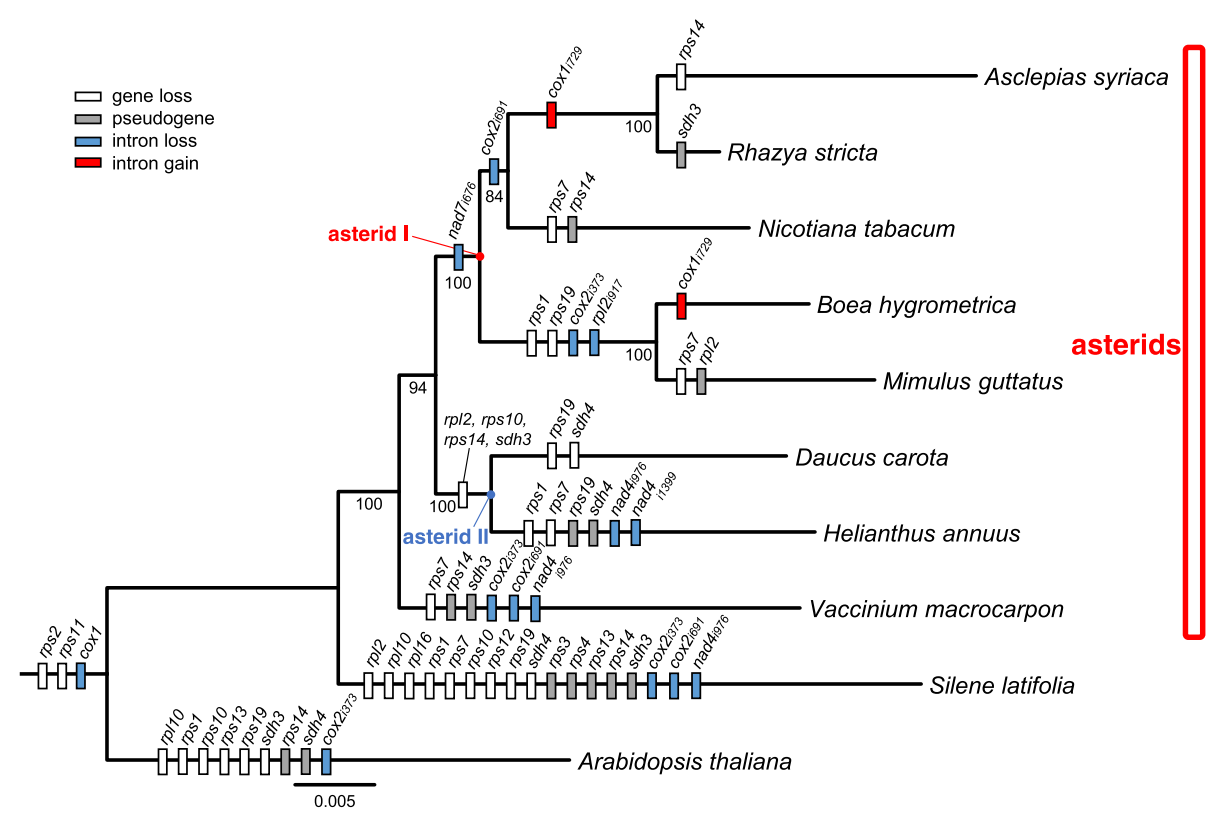

Figure 8 Phylogenetic distribution of gene/intron content (loss and gain) among eight asterids and two outgroups. The tree was inferred using the maximum likelihood (ML) method with the 'GTRGAMMA' evolutionary model under the rapid bootstrap algorithm (1000 replicates). Bootstrap support values $>50 \%$ are shown at nodes. The 24 genes include $\operatorname{atp}[1,4,6,8,9], \operatorname{ccm}[B, C, F C$, Fn], cob, cox[1-3], matR, mttB, and nad[1, 2, 3, 4, 4L, 5, 6, 7, 9]. The cox1 introns have been lost in the ancestor of angiosperms.

other protein genes (Tables 2 and 3; Additional file 1: Table S11).

All species in the asterid I lineage were missing the intron nad7i676. Rhazya, Asclepias and Nicotiana share the loss of the intron cox2i691, and Boea and Mimulus share the loss of two genes (rps1 and rps19) and two introns (cox2i373 and rpl2i917). The phylogenetic distribution of the intron cox1i729 (Figure 8) indicates that the gain of this intron occurred independently in the Rhazya/Asclepias clade and Boea. Among the asterid I species analyzed, the presence of $s d h 3$ as a pseudogene was unique to Rhazya. Two species, Helianthus and Vaccinium, in the asterid II lineage share the loss of four genes ( $r p l 2$, rps10, rps14, and sdh3). No shared losses/gains were found for the asterid clade relative to the two outgroups.

BlastN searches of the (R)-mandelonitrile lyase gene, which is represented by two overlapping ORFs in Rhazya (Additional file 2: Figure S3), were performed against asterid mitochondrial genomes. Six additional asterids, Asclepias (66 bp), Boea (193 bp), Daucus (520 bp), Helianthus (273 bp), Nicotiana (495 bp), and Vaccinium (246 bp) contain fragments of this nuclear gene (Additional file 2: Figure S3B).

\section{Discussion}

\section{Organization of Rhazya stricta organelle genomes}

The $R$. stricta plastid genome is highly conserved with gene content and gene order identical to the ancestral genome organization of angiosperms [14,37]. The size of
R. stricta plastid genome at $154,841 \mathrm{bp}$ is close to the median genome size for photosynthetic land plants [38]. A recent study found that the plastid genome of Asclepias contains a $2.4 \mathrm{~kb}$ segment of mitochondrial DNA with an rpl2 pseudogene, and this transfer has been documented in other genera of the tribe Asclepiadeae (subfamily Asclepiadoideae, Apocynaceae) [39]. The Rhazya plastid genome does not contain any mitochondrial-like sequences, confirming that transfer of mitochondrial DNA into the plastid genome is restricted to a single tribe of Apocynaceae. Therefore, inter-compartmental transfers between the Rhazya plastid and mitochondrial genomes have occurred in one direction only.

The $R$. stricta mitochondrial genome exhibits several complex evolutionary features, including a dynamic genome structure that has been shaped by repeat families and intramolecular recombination, invasion of the genome by both plastid and nuclear sequences, and the putative functional transfer of two genes to the nucleus. Recombination between repeats has resulted in a master chromosome and multiple subgenomic circles [19-22,40]. The R. stricta mitochondrial genome contains seven repeat families, including two large and five intermediate-sized repeats. These repeats are involved in homologous recombination in the Rhazya mitochondrial genome. Two distinct conformations of the master chromosome and six subgenomic circles have been confirmed by PCR (Additional file 2: Figures S1 and S2), however additional chromosome configurations may be present in Rhazya mitochondria. 
Table 2 Rhazya protein-coding gene content compared to other asterids and two representative angiosperms

\begin{tabular}{|c|c|c|c|c|c|c|c|c|c|c|}
\hline & Rhazya & Asclepias & Nicotiana & Mimulus & Boea & Daucus & Helianthus & Vaccinium & Silene & Arabidopsis \\
\hline $\operatorname{atp}[x]$ & $\bullet$ & $\bullet^{a}$ & $\bullet$ & $\bullet$ & $\bullet$ & $\bullet$ & $\bullet$ & $\bullet^{d}$ & $\bullet$ & $\bullet$ \\
\hline $\operatorname{ccm}[x]$ & $\bullet$ & $\bullet$ & $\bullet$ & $\bullet$ & $\bullet$ & $\bullet$ & $\bullet$ & $\bullet$ & $\bullet$ & $\bullet$ \\
\hline$c o b$ & $\bullet$ & $\bullet$ & $\bullet$ & $\bullet$ & $\bullet$ & $\bullet$ & $\bullet$ & $\bullet^{d}$ & $\bullet$ & $\bullet$ \\
\hline $\operatorname{cox}[x]$ & $\bullet$ & $\bullet$ & $\bullet$ & $\bullet$ & $\bullet$ & $\bullet$ & $\bullet^{c}$ & $\bullet$ & $\bullet$ & $\bullet$ \\
\hline matR & $\bullet$ & $\bullet$ & $\bullet$ & $\bullet$ & $\bullet$ & $\bullet$ & $\bullet$ & $\bullet$ & $\bullet$ & $\bullet$ \\
\hline$m t t B$ & $\bullet$ & $\bullet$ & $\bullet$ & $\bullet$ & $\bullet^{b}$ & $\bullet$ & $\bullet^{c}$ & - & - & $\bullet$ \\
\hline $\operatorname{nad}[x]$ & $\bullet$ & $\bullet$ & $\bullet$ & $\bullet$ & $\bullet$ & $\bullet$ & $\bullet^{c}$ & $\bullet^{d}$ & • & $\bullet$ \\
\hline$r p / 2$ & $\bullet$ & $\bullet$ & $\bullet$ & $\psi$ & $\bullet^{b}$ & $\circ$ & $\circ$ & $\bullet$ & ○ & $\bullet$ \\
\hline$r p / 5$ & $\bullet$ & $\bullet$ & $\bullet$ & $\bullet$ & $\bullet^{b}$ & $\bullet$ & • & - & • & • \\
\hline$r p / 10$ & $\bullet$ & $\bullet$ & $\bullet$ & $\bullet$ & $\bullet^{b}$ & $\bullet$ & $\bullet$ & $\bullet$ & $\circ$ & $\circ$ \\
\hline$r p / 16$ & $\bullet$ & $\bullet^{a}$ & $\bullet$ & $\bullet$ & $\bullet$ & $\bullet$ & $\bullet$ & $\bullet$ & $\circ$ & $\bullet$ \\
\hline rps1 & $\bullet$ & $\bullet$ & $\bullet$ & $\circ$ & $\circ$ & $\bullet$ & $\circ$ & $\bullet$ & $\circ$ & $\circ$ \\
\hline rps2 & $\circ$ & $\circ$ & $\circ$ & $\circ$ & $\circ$ & $\circ$ & $\circ$ & $\circ$ & $\circ$ & $\circ$ \\
\hline rps3 & $\bullet$ & $\bullet$ & $\bullet$ & $\bullet$ & $\bullet$ & $\bullet$ & $\bullet^{c}$ & $\bullet^{d}$ & $\psi$ & $\bullet$ \\
\hline rps4 & $\bullet$ & $\bullet$ & $\bullet$ & $\bullet$ & $\bullet$ & $\bullet$ & $\bullet$ & $\bullet$ & $\psi$ & $\bullet$ \\
\hline rps7 & $\bullet$ & $\bullet$ & $\circ$ & $\circ$ & $\bullet$ & $\bullet$ & $\circ$ & $\circ$ & $\circ$ & $\bullet$ \\
\hline $\operatorname{rps} 10$ & $\bullet$ & $\bullet$ & $\bullet$ & $\bullet$ & $\bullet^{b}$ & $\circ$ & $\circ$ & $\bullet$ & $\circ$ & $\circ$ \\
\hline rps 11 & $\circ$ & $\circ$ & $\circ$ & $\circ$ & $\circ$ & $\circ$ & $\circ$ & $\circ$ & $\circ$ & ० \\
\hline $\operatorname{rps} 12$ & $\bullet$ & $\bullet$ & $\bullet$ & $\bullet$ & $\bullet$ & $\bullet$ & $\bullet$ & $\bullet$ & $\circ$ & $\bullet$ \\
\hline $\operatorname{rps} 13$ & $\bullet$ & $\bullet$ & $\bullet$ & $\bullet$ & $\bullet$ & $\bullet$ & $\bullet$ & $\bullet$ & $\psi$ & $\circ$ \\
\hline rps 14 & $\bullet$ & $\circ$ & $\psi$ & $\bullet$ & $\bullet b$ & $\circ$ & $\circ$ & $\psi$ & $\psi$ & $\psi$ \\
\hline rps 19 & $\bullet$ & $\bullet$ & $\bullet$ & $\circ$ & $\circ$ & $\circ$ & $\psi^{c}$ & - & $\circ$ & $\circ$ \\
\hline sdh3 & $\psi$ & $\bullet$ & $\bullet$ & $\bullet$ & $\bullet$ & $\circ$ & $\circ$ & $\psi$ & $\psi$ & ० \\
\hline sdh4 & $\bullet$ & $\bullet$ & $\bullet$ & $\bullet$ & $\bullet$ & $\circ$ & $\psi^{c}$ & $\bullet$ & $\circ$ & $\psi$ \\
\hline - present & 38 & 38 & 37 & 35 & 37 & 33 & 31 & 36 & 25 & 31 \\
\hline o absent & 2 & 3 & 3 & 5 & 4 & 8 & 8 & 3 & 11 & 8 \\
\hline$\Psi$ pseudo & 1 & 0 & 1 & 1 & 0 & 0 & 2 & 2 & 5 & 2 \\
\hline
\end{tabular}

${ }^{\mathrm{a} A l t h o u g h}$ atp6 and rp/16 of Asclepias syriaca are annotated in GenBank as pseudogenes, new analyses suggest functionality for both genes. ${ }^{\mathrm{b}}$ Boea hygrometrica, ${ }^{\mathrm{C}}$ Helianthus annuus, and ${ }^{d}$ Vaccinium macrocarpon mitochondrial genomes were re-annotated using MITOFY $[27]$ in this $\operatorname{study.~atp}[x]=a t p[1,4,6,8,9], c c m[x]=c \mathrm{~cm}[B$, $C, F C, F n], \operatorname{cox}[x]=\operatorname{cox}[1-3], \operatorname{nad}[x]=\operatorname{nad}[1,2,3,4,4 L, 5,6,7,9]$.

PCR recombination has been suggested as a complicating factor in utilizing this approach to confirm recombination among repeats in plant mitochondrial genomes [21]. However, the use of three different Illumina libraries (one paired end and two mate pair), the high depth of coverage (average coverage is 679X), and corrected PacBio data provide additional confidence in the PCR results. Recombination among the repeats may have influenced gene content by facilitating gene fusion and pseudogenization events [41]. For example, most mitochondrial genes of Rhazya are highly conserved in length compared to other angiosperms except atp6. This gene usually contains extended sequences at the $\mathrm{N}$-terminus that are highly divergent across angiosperms even within species [42]. In most cases, atp 6 is located at the border of repeat regions, suggesting that this gene is frequently involved in genomic recombination [42]. In Rhazya only the atp6 conserved domain is present and is located downstream from repeat 2 (R2; Additional file 2: Figure S4B). The $\mathrm{N}$-terminal extended sequences of atp6 in Rhazya may have been cleaved by genomic recombination activities upstream of the protein gene, but remnants of these extended sequences were not detected due to high sequence divergence and frequent recombination activities. Two ORFs (318 and 324) located within repeat families including repeat 2 have one of the diagnostic features of chimeric genes: the presence of a transmembrane domain [24] (Additional file 2: Figure S4B). Studies have shown that chimeric ORFs containing a portion of ATP synthase subunit are frequently associated with rearrangements in plant mitochondrial genomes [22,24]. The situation in Rhazya supports a connection between ATP synthase subunits and recombination in mitochondrial genomes. 
Table 3 Rhazya intron content compared to other asterids and two representative angiosperms

\begin{tabular}{|c|c|c|c|c|c|c|c|c|c|c|}
\hline & Rhazya & Asclepias & Nicotiana & Mimulus & Boea & Daucus & Helianthus & Vaccinium & Silene & Arabidopsis \\
\hline ccmFcis29 & $\bullet$ & $\bullet$ & $\bullet$ & $\bullet$ & $\bullet$ & $\bullet$ & $\bullet^{\mathrm{b}}$ & $\bullet$ & $\bullet$ & $\bullet$ \\
\hline $\operatorname{cox} 11729$ & - & - & $\circ$ & $\circ$ & - & $\circ$ & $\circ$ & $\circ$ & $\circ$ & o \\
\hline $\cos 2 i 373$ & $\bullet$ & $\bullet$ & $\bullet$ & $\circ$ & $\circ$ & $\bullet$ & $\bullet$ & $\circ$ & $\circ$ & $\circ$ \\
\hline cox2i691 & $\circ$ & $\circ$ & ○ & $\bullet$ & $\bullet$ & $\bullet$ & $\bullet$ & $\circ$ & $\circ$ & $\bullet$ \\
\hline nad1i394 & $\square$ & $\square$ & $\square$ & $\square$ & $\square$ & $\square$ & $\square^{\mathrm{b}}$ & $\square$ & $\square$ & $\square$ \\
\hline nadli477 & $\bullet$ & $\bullet$ & $\bullet$ & $\bullet$ & $\bullet$ & $\bullet$ & $\bullet^{\mathrm{b}}$ & $\bullet$ & • & $\bullet$ \\
\hline nad1i669 & $\square$ & $\square$ & $\square$ & $\square$ & $\square$ & $\square$ & $\square^{\mathrm{b}}$ & $\square$ & $\square$ & $\square$ \\
\hline nadli728 & $\square$ & $\square$ & $\square$ & $\square$ & $\square$ & $\square$ & $\square^{\mathrm{b}}$ & $\square$ & $\square$ & • \\
\hline $\operatorname{nad} 2 i 156$ & $\bullet$ & $\bullet$ & $\bullet$ & $\bullet$ & $\bullet$ & $\bullet$ & $\bullet^{b}$ & $\bullet$ & $\bullet$ & $\bullet$ \\
\hline $\operatorname{nad} 2 i 542$ & $\square$ & $\square$ & $\square$ & $\square$ & $\square$ & $\square$ & $\square^{b}$ & $\square$ & $\square$ & $\square$ \\
\hline nad2i709 & $\bullet$ & $\bullet$ & $\bullet$ & $\bullet$ & $\bullet$ & $\bullet$ & $\bullet^{b}$ & $\bullet$ & • & $\bullet$ \\
\hline nad2i1282 & - & $\bullet$ & $\bullet$ & $\bullet$ & $\bullet$ & $\bullet$ & $\bullet^{b}$ & $\bullet$ & • & - \\
\hline nad4i461 & - & $\bullet$ & $\bullet$ & $\bullet$ & $\bullet$ & $\bullet$ & $\bullet^{b}$ & $\bullet$ & • & - \\
\hline nad4i976 & - & $\bullet$ & • & • & $\bullet$ & $\bullet$ & $\mathrm{o}^{\mathrm{b}}$ & ○ & $\circ$ & • \\
\hline nad4i1399 & $\bullet$ & $\bullet$ & $\bullet$ & $\bullet$ & $\bullet$ & $\bullet$ & $o^{b}$ & $\bullet$ & $\bullet$ & $\bullet$ \\
\hline nad5i230 & $\bullet$ & $\bullet$ & $\bullet$ & $\bullet$ & $\bullet$ & $\bullet$ & $\bullet$ & $\bullet$ & $\bullet$ & $\bullet$ \\
\hline nad5i1455 & $\square$ & $\square$ & $\square$ & $\square$ & $\square$ & $\square$ & $\square^{b}$ & 口 & $\square$ & $\square$ \\
\hline nad5i1477 & $\square$ & $\square$ & $\square$ & $\square$ & 口 & $\square$ & $\square^{b}$ & 口 & $\square$ & $\square$ \\
\hline nad5i1872 & • & • & • & • & $\bullet$ & $\bullet$ & $\bullet^{b}$ & • & • & • \\
\hline nad7i140 & • & • & • & • & • & • & $\bullet^{b}$ & • & • & • \\
\hline nad7i209 & • & • & • & • & • & • & $\bullet^{\mathrm{b}}$ & • & • & • \\
\hline nad7i676 & $\circ$ & $\circ$ & $\circ$ & $\circ$ & $\circ$ & • & $\bullet^{b}$ & • & • & • \\
\hline nad7ig17 & • & • & • & • & • & • & $\bullet^{\mathrm{b}}$ & • & • & • \\
\hline rp/2i917 & • & • & • & $\circ$ & $\circ^{a}$ & $x$ & $x$ & - & $x$ & • \\
\hline rps10i235 & - & • & • & • & $\bullet^{\mathrm{a}}$ & x & $x$ & $\circ$ & $x$ & $x$ \\
\hline rps3i74 & - & - & - & - & - & - & $\bullet$ & •c & - & - \\
\hline
\end{tabular}

-; cis-spliced, $\square$; trans-spliced, $\circ$; intron absent, $\mathrm{x}$; gene loss. The genes of ${ }^{\mathrm{a}}$ Boea hygrometrica and ${ }^{\mathrm{b}}$ Helianthus annuus and ${ }^{\mathrm{C}}$ Vaccinium macrocarpon mitochondrial genomes were re-annotated using MITOFY [27] in this study.

Twelve other potential chimeric ORFs are present in the $R$. stricta mitochondrial genome. One of these, ORF56b, has features of CMS [24], including fragments containing a portion of three mitochondrial genes $(r p l 2$, matR, and $c c m F n$ ), transmembrane helices and overlap with a second copy of repeat 6 (R6; Additional file 2: Figure S4A). Further work is needed to determine if these other ORFs are functional and associated with genomic recombination.

Plant mitochondrial genomes typically contain DNA originating from plastid and nuclear genomes, and in some cases from other species including bacteria, viruses and plants [25-29]. The Rhazya mitochondrial genome contains considerable foreign DNA, accounting for at least $8.9 \%$ of the genome. Plastid-derived sequences are variable in mitochondrial genomes of seed plants and account for $1-12 \%$ [17] indicating that Rhazya has an intermediate amount in its genome (6\%). The inserted plastid sequences of Rhazya include full-length protein- coding and tRNA genes, most of which are presumed nonfunctional, but some tRNA genes may be candidates for functional transfer as reported for wheat and potato $[43,44]$. The absence of trnM-CAU in Rhazya is unusual among sequenced angiosperm mitochondrial genomes as previous phylogenetic analyses have suggested that transfer of trnM-CAU to the mitochondrion occurred in the common ancestor of extant angiosperms $[45,46]$.

The Rhazya mitochondrial genome also contains numerous nuclear-derived sequences, most of which are transposable elements (TEs) (Figure 6A and 6B; Additional file 1: Table S3). Most of the TEs are located in intergenic spacers but 14 are found in genes (Additional file 1: Table S4). Previous studies have only observed TEs in intergenic spacers reviewed in [17] but in some cases searches for TEs were limited to these regions see reference [27]. In addition to TEs, the Rhazya mitochondrial genome contains two sequences homologous to the nuclear gene encoding ( $R$ )-mandelonitrile lyase. One 
copy of the sequence is nearly complete and the presence of a transcript with $100 \%$ sequence identity to this copy suggests that it is expressed. Previous studies have shown that some angiosperm mitochondrial genomes contain fragments of nuclear protein-coding genes $[21,27,47]$, however the assembled transcriptome contig and RT-PCR result refute a functional role for this gene in Rhazya mitochondria. The presence of internal stop codons in the transcript suggests that it is likely a product of relaxed transcription in mitochondria [48].

The Rhazya mitochondrial genome has acquired the intron cox1i729, which is commonly the subject of horizontal transfer across angiosperms [49]. This intron in Rhazya is highly similar (96.7-98.8\% sequence identity) to introns from eight other genera of Apocynaceae. Sanchez-Puerta et al. [49] suggested six independent gains of this intron in the Apocynaceae. Two alternative hypotheses for the cox 1 intron were suggested: stochastic loss and horizontal transfer [50,51]. The dynamics of cox1 intron gain or loss within the Apocynaceae requires further study to better understand its evolutionary history in the family.

\section{Mitochondrial gene transfer in Rhazya genomes}

Rhazya contains 38 of the 41 protein-coding genes that are found in the ancestral angiosperm mitochondrial genome [17]. Two ribosomal proteins, rps2 and rps11, that are absent in Rhazya have been lost from the mitochondrial genomes of nearly all core eudicots [52]. The third gene, $s d h 3$, has been lost numerous times across angiosperms [52] and appears to be a pseudogene in Rhazya. Analyses of the transcriptome and a draft nuclear genome of Rhazya facilitated identification of putative functional copies of $s d h 3$ and rps14 in the nucleus.

Rhazya sdh3 has been transferred to the nucleus and has acquired a mitochondrial targeting presequence as well as one intron and a portion of chaperonin gene $h s p 22$ (Figure 5). Previous studies have identified 14 independent transfers of $s d h 3$ to the nucleus in angiosperms, and in all cases mitochondrial targeting presequences were acquired from preexisting nuclear genes [36,53]. A previous study reported putative functional transfers of $s d h 3$ in five other asterid families, Asteraceae, Convolvulaceae, Ericaceae, Lamiaceae and Orobanchaceae [36]. This previous report, combined with the phylogenetic distribution of $s d h 3$ loss from the mitochondrial genomes of sequenced asterid genomes (Figure 8), indicates that this gene has been transferred to the nucleus multiple times in this clade.

In case of the Rhazya rps14, two copies were identified in the nucleus. One copy has a mitochondrial targeting presequence and is likely functional while the second copy is likely non-functional considering its level of divergence from the putative functional copy (Figures 3 and 4A). There are at least three alternative explanations for the origin of two nuclear copies: 1) rps14 was transferred to the nucleus and after acquiring the mitochondrial targeting presequence it was duplicated, followed by the loss of the targeting presequence in one copy; 2) the gene was duplicated after transfer and only one copy acquired a targeting presequence; or 3) there were two independent transfers of rps14 to the nucleus and only one of the copies acquired a targeting presequence. Phylogenetic analyses support a single transfer of rps14 to the nucleus (Figure 4A) but it is not possible with available data to discern between alternatives one and two. A previous study showed that the rps14 coding sequence has been transferred to the nucleus independently at least three times in grasses and that mitochondrial rps14 pseudogene transcripts are expressed [54], although this may be due to the well-known phenomenon of relaxed transcription in mitochondria [48]. Although Rhazya mitochondrial rps14 has a deletion at the 3 ' end causing a frameshift mutation, the gene may retain functionality as it is nearly full length relative to other angiosperms (Figure 3B and 3C). Moreover, the mitochondrial copy lacks nonsense mutations and transcriptome data showed that the mitochondrial rps 14 is co-transcribed with $r p l 5$ (Figure $3 \mathrm{~A}$ ) as a reported for tobacco [19]. Following transfers to the nucleus, the co-existence of putative functional nuclear and mitochondrial gene copies has been suggested for only three genes among angiosperms, including cox2 in some Fabaceae [55], rpl5 in Triticum [56], and sdh4 in Populus [57].

\section{Evolutionary comparisons among asterid mitochondrial genomes}

Comparing the mitochondrial genome sequences of Rha$z y a$ with the seven other asterids and two representative non-asterids provides insight into the distinct evolutionary events that have occurred across this clade. Genome sizes vary 2.4-fold among the asterids and the Rhazya genome represents an intermediate size within the asterid I lineage (Figure 6A). No clear correlation is seen between repetitive DNA or TE content and genome size among asterid mitochondrial genomes, whereas the amount of transferred plastid DNA tends to scale with genome size. For example, repetitive DNA content is highest in Rhazya, but Asclepias is the largest genome despite its 9.6-fold lower repetitive DNA content than Rhazya (Figure 6A). Likewise, although Daucus has the second highest repetitive DNA content, it is the smallest genome among the eight asterids (Figure 6A). The asterid mitochondrial genomes also differ in the structure and complexity of their repeats. Rhazya, Nicotiana, Mimulus, Helianthus and Daucus have large repeats, in contrast to the other asterids. These genomes display diverse patterns of repeats that appear to facilitate recombination and can range in size from short (124 bp) to long (36 kb) within a single species.

There are very few colinear clusters of genes across the eight asterid mitochondrial genomes, which likely 
reflects the very different patterns of repeats that have caused rearrangements (Figure 7). Overall, this comparison indicates that asterid mitochondrial genomes have contrasting evolutionary histories, resulting in very diverse organization and gene content. A better understanding of the evolutionary history of recombination among asterid mitochondrial genomes requires genome sequences for more taxa and comparisons of nuclear genes encoding DNA repair and recombination proteins including RecA-like recombinases, MutS homologue 1, the Whirlies and other organellar single stranded DNAbinding proteins that have been implicated in maintenance of genome stability [58].

The phylogenetic distribution of gene and intron losses and TE insertions revealed some shared and many independent events across asterids (Figure 8, Additional file 1: Table S4). Most mitochondrial gene loss/pseudogenization events involve ribosomal proteins and $s d h$ genes, as in other angiosperms [52]. The two proteincoding genes rps 2 and rps 11 were lost in the ancestor of eudicots [52]. The presence of the intron coxli729 in the Rhazya/Asclepias clade and Boea suggests that it was gained independently twice in the asterid clade since this intron was lost in the ancestor of angiosperms [49]. The asterid I lineage shares the loss of the intron nad7i676, indicating that this loss occurred in the common ancestor of the clade. The loss of the intron cox2i691 in Rhazya, Asclepias and Nicotiana, and the loss of two genes (rps1 and rps19) and two introns (cox2i373 and rpl2i917) in Boea and Mimulus indicate that these events occurred in the most recent common ancestor of each clade. In case of the Mimulus rpl2, the phylogenetic distribution suggests that this gene became pseudogenized after the intron was lost in common ancestor of the Boea/Mimulus clade. The asterid II clade has lost four genes, three ribosomal proteins genes and one $s d h$ gene. The phylogenetic distribution of rps14 among asterids suggests that this gene was transferred to the nucleus in the common ancestor of the Rhazya, Asclepias and Nicotiana clade. The fate of the gene was different in the three species; it has been lost in Asclepias, pseudogenized in $\mathrm{Ni}$ cotiana while both the mitochondrial and nuclear copies are likely functional in Rhazya. The duplication event of nuclear rps14 within the asterid I clade is likely more complicated and will require more nuclear genome data to resolve. There are a number of shared gains of TEs within genic regions across asterids (Additional file 1: Table S4). All asterids examined share the same TEs in the same location except for the TE in the cox3 gene. This suggests that nearly all TEs were transferred in the ancestor of the asterid clade. The cox3 TE insertion in Rhazya and Nicotiana differ in size (86 versus $41 \mathrm{bp}$ ) and TE class (LTR/Copia in Rhazya and DNA/MuDR in Nicotiana).

\section{Conclusions}

Organelle genomes of Rhazya stricta, a member of the asterid I clade, provide important information for improving the understanding of mitochondrial genome evolution among angiosperms. The mitochondrial genome exhibits a number of phenomena that have been observed in other species [17], including the presence of recombinogenic repeats that generate a multipartite organization with a master chromosome and subgenomic circles, a high incidence of transferred DNA from the plastid and nuclear genomes, and gene transfers from the mitochondrion to the nucleus. The organellar genomic sequences, combined with nuclear transcriptome and genome data, have enabled a rigorous examination of these events. Rhazya is unique among the eight sequenced asterids in the types of events that have shaped the evolution of its mitochondrial genome. The organelle genomes of $R$. stricta provide valuable genomic resources for utilizing this important medicinal plant in biotechnology applications.

\section{Methods}

\section{Plant material}

Rhazya stricta seeds were obtained from natural populations collected in the Makkah Province, Saudi Arabia. Seeds were soaked in water overnight at $37^{\circ} \mathrm{C}$ then transferred to Profile ${ }^{\circ}$ Field \& Fairway $^{\mathrm{mi}}$ inorganic ceramic particles (Buffalo Grove, IL) in a growth chamber $\left(16 \mathrm{~h}\right.$ light, $8 \mathrm{~h}$ dark, $\left.38^{\circ} \mathrm{C}\right)$ for germination. Young leaves were flash frozen in liquid nitrogen for DNA and RNA isolation and stored at $-80^{\circ} \mathrm{C}$.

\section{DNA isolation}

Genomic DNA isolation was performed as described by Doyle and Doyle [59] with modifications. Cetyl trimethylammonium bromide buffer was augmented with $3 \%$ polyvinylpyrrolidone and 3\% beta-mercaptoethanol (Sigma, St. Louis, MO). Organic phase separation was repeated until the aqueous fraction was clear. DNA pellets were resuspended in $\sim 200 \mu \mathrm{L}$ DNase-free water. Following treatment with RNase A (ThermoScientific, Lafayette, CO) samples were again subjected to phase separation with chloroform, and DNA was recovered by ethanol precipitation. Samples were resuspended in DNase-free water, evaluated for intactness and concentration by gel electrophoresis and stored at $-20^{\circ} \mathrm{C}$.

\section{DNA sequencing and genome assembly}

Genomic DNA was used to construct three Illumina libraries: a paired-end (PE) fragment library with an average insert size of $626 \mathrm{bp}$, and two mate pair (MP) libraries with an average insert sizes of 2,363 and $4,340 \mathrm{bp}$. Sequence reads were generated using the HiSeq 2000 sequencing platform (Illumina, San Diego, 
CA) at the Genome and Sequence Analysis Facility (GSAF) at the University of Texas at Austin. A $10 \mathrm{~kb}$ SMRT cell library was constructed for PacBio RS II sequencing (Pacific Biosciences, Menlo Park, CA) and eight SMRT cells of sequence data were generated at the University of Florida Interdisciplinary Center for Biotechnology Research. All PacBio reads were corrected using PacBioToCA [60] with 634 Mb (10\%) of PE Illumina reads.

To complete the plastid genome, the PE Illumina reads were assembled de novo with Velvet v.1.2.08 [61] using multiple $k$-mers. For the mitochondrial genome, Illumina reads were assembled de novo with Velvet using multiple $k$-mers values and by separately combining the PE reads with each of the two MP read sets. The parameters were modified according to the depth of the read coverage of each organelle genome. The initial plastid contigs were assembled in Geneious R6 v.6.1.6 [62], which was also used to visualize and finish the mitochondrial genome assembly by tracking and end inspection of the initial mitochondrial contigs overlapping with corrected PacBio reads. The corrected PacBio reads were further used to fill gaps and to validate regions of plastid integration in the mitochondrial genome. The correction of PacBio reads and the de novo genome assemblies were performed on Lonestar Dell Linux Cluster of the Texas Advanced Computing Center (TACC).

\section{Genome annotations and analyses}

The plastid and mitochondrial genomes were annotated using DOGMA [63] and MITOFY [27], respectively. Intron nomenclature for mitochondrial genes follows Dombrovska and Qiu [64]. All tRNA genes were predicted using tRNAscan-SE v.1.3.1 [65]. Sequences of the annotated organelle genomes were deposited in GenBank (accession numbers KJ485849 and KJ485850). Genome maps were drawn with OGDRAW [66].

Repetitive sequences were identified by performing BlastN v.2.2.28+ comparisons of the Rhazya mitochondrial genome against itself with an e-value cutoff of 1e-10 and at least $90 \%$ sequence identity. Genome maps were drawn with Circos v.0.64 [67]. Repeat regions involved in recombination were identified by mapping of the corrected PacBio reads. To test for alternative recombinogenic conformations of the mitochondrial genome, polymerase chain reaction (PCR) was carried out using total genomic DNA and primers designed by Primer3 [68] in Geneious R6 (Additional file 1: Table S12).

Open Reading Frames (ORFs) longer than 300 bp in Rhazya mitochondrial genome were analyzed using the ORF finder from the National Center for Biotechnology Information (NCBI) [69]. Any ORFs that overlapped with annotated Rhazya mitochondrial genes and genes transferred from the plastid were excluded. To search for chimeric ORFs, all ORFs longer than $150 \mathrm{bp}$ were compared with annotated Rhazya mitochondrial genes using BlastN with an e-value cutoff of 1e-3, minimum length of $30 \mathrm{bp}$ (as described in Mower et al. [22]) and at least $90 \%$ sequence identity. Transmembrane helices in detected ORFs were predicted using TMHMM Server v.2.0 [70].

Plastid-like sequences transferred to the mitochondrial genome were identified by performing BlastN searches of Rhazya plastid genome against the mitochondrial genome in Geneious R6 with an e-value cutoff of 1e-10, at least $80 \%$ sequence identity and minimum length of $50 \mathrm{bp}$. Mitochondrial-encoded genes (CDS) were used as BlastN queries against the Rhazya plastid genome to search for gene sequences transferred into the plastid genome. To identify putative transposable elements (TEs), the mitochondrial genome was searched against CENSOR web server [71] with default parameters and 'green plants' as a reference sequence source. In addition to the Rhazya mitochondrial genome, seven other asterid mitochondrial genomes (Asclepias syriaca; NC_022796, Boea hygrometrica; NC_002511, Daucus carota subsp. sativus; NC_ 017855, Helianthus annuus; NC_023337, Mimulus guttatus; NC_018041, Nicotiana tabacum; NC_006581, and Vaccinium macrocarpon; NC_023338) were examined for repetitive sequences, plastid-like sequences and TEs. To search for plastid-like sequences in mitochondrial genomes, their plastid counterparts (Asclepias syriaca; $\mathrm{NC}_{-}$ 022432, Boea hygrometrica; NC_016468, Daucus carota subsp. sativus; NC_008325, Helianthus annuus; $\mathrm{NC}_{-}$ 007977, Jasminum nudiflorum [for Mimulus guttatus]; NC_008407, Nicotiana tabacum; NC_001879, and Vaccinium macrocarpon; NC_019616) were used.

RNA editing sites were predicted using PREP-Mt [72] with a cutoff value of 0.5 and PREPACT v.2.12.2 [73] using default settings.

\section{RNA isolation, transcriptome sequencing, and RT-PCR}

Total RNA isolation, library construction and Illumina sequencing were performed according to Zhang et al. [74]. Duplex specific nuclease normalization (Evrogen, Moscow, Russia) of the RNA samples, Illumina RNAseq library construction and sequencing were carried out at the GSAF. Raw read output from $R$. stricta RNAseq was deposited in the small read archive (SRA) at the NCBI (accession number SRR1151604).

To confirm a putative transfer of the nuclear gene (R)mandelonitrile lyase to the mitochondrion, reverse transcription was performed with gene-specific primers using ImProm-II ${ }^{\text {Tm }}$ Reverse Transcriptase (Promega, USA). PCR amplification was done with primer pairs specific to the ORFs (Additional file 1: Table S12), the products were treated with ExoSAP-IT (New England Biolabs, Ipswich, 
MA) and Sanger sequenced at the University of Texas Institute of Cellular and Molecular Biology core facility.

\section{Identification of genes transferred to the nuclear genome}

Rhazya de novo transcriptome assembly was performed using Trinity [75], released on 2013-02-25 with the script used in Zhang et al. [74] at TACC. The transferred genes were sought using BlastN (e-value cutoff of 1e-10) of the 38 Rhazya mitochondrial-encoded genes and the pseudogene of $s d h 3$ against the transcriptome contigs. TargetP v.1.1 [76] and Predotar v.1.03 [77] were used to predict mitochondrial targeting presequences (mTP). Putative ORFs were searched using Phytozome v.9.1 [78] with BLASTX and 'asterid' as a reference sequence source to identify plant gene families. The NCBI Conserved Domain Database (CDD) was used for functional domain annotation [79]. Nucleotide and amino acid sequences of nuclear and mitochondrial genes were aligned with MUSCLE [80] in Geneious R6.

\section{Phylogenetic analysis of $r p s 14$ and $s d h 3$ genes}

Phylogenetic analyses were performed on data sets of two genes transferred to the nucleus, rps 14 and sdh3. Each data set included mitochondrial and nuclear copies of both genes (Additional file 1: Table S13), and the data sets were aligned with MUSCLE [80] in Geneious R6. Maximum likelihood phylogenetic trees were constructed using RAxML v.7.2.8 [81] with the 'GTRGAMMA' evolutionary model under the rapid bootstrap algorithm with 1000 replicates at TACC.

\section{Availability of supporting data}

All supporting data is included as additional files. Complete mitochondrial and plastid genome sequences have been submitted to GenBank (accession numbers KJ485849 and KJ485850) and raw sequencing reads from RNAseq were deposited in the small read archive (SRA) at the NCBI (accession number SRR1151604).

\section{Additional files}

Additional file 1:Table S1. The gene content of the Rhazya stricto mitochondrial genome. Table S2. Predicted repeat pairs in the Rhazya mitochondrial genome. Table S3. Putative transposable elements (TEs) in the Rhazya mitochondrial genome. Table S4. Fourteen putative transposable elements (TEs) located in the genic regions. Table S5. Blast results of ORFs (>300 bp) in the Rhazya mitochondrial genome. Table S6. Potential chimeric ORFs. Table S7. Blast result of plastid-derived DNA segments in the mitochondrial genome of Rhazya stricta. Table $\mathbf{S 8 .}$ Predicted RNA editing in 38 protein-coding genes for the Rhazya mitochondrial genome. Table S9. RNA editing validation of 11 genes using transcriptome data. Table S10. Genome size, GC content, repetitive DNA, plastid-like DNA and transposable elements in eight asterid mitochondrial genomes. Table S11. Rhazya rRNA and tRNA content compared to other asterids and two representative angiosperms. Table S12. Primers used for testing alternative recombinogenic conformations and for confirming ORFs that represented the (R)-mandelonitrile lyase gene in the Rhazya mitochondrial genome. Table S13. Information on the phylogenetic analyses and alignment of the rps 14 and sdh 3 genes.

Additional file 2: Figure S1. Seven families of repeats (R1-R7) of Rhazya mitochondrial genome involved in recombination. Figure S2. PCR strategy for identifying intramolecular recombination across mitochondrial repeats. Figure S3. The two mitochondrial ORFs representing the nuclear (R)-mandelonitrile lyase sequence [82]. Figure S4. Potential chimeric ORFs. Figure S5. Conserved mitochondrial gene blocks among eight asterids.

\section{Competing interests}

The authors declare that they have no competing interests.

\section{Authors' contributions}

SP assembled, finished, and annotated mitochondrial and plastid genomes, performed RT-PCR and all data analyses, submitted sequences to NCBI, and wrote the first draft of all sections of the manuscript except DNA and RNA isolation; TAR assisted in collecting seeds of Rhazya stricta, germinated seeds and cared for plants in the greenhouse, performed DNA and RNA isolations, prepared samples for genome and transcriptome sequencing, drafted DNA and RNA methods section, and edited drafts of the manuscript; JSMS contributed to the design of the project, assisted in collecting seeds of Rhazya stricta, and read/edited the manuscript; MHZM contributed to the design of the project, assisted in collecting seeds of Rhazya stricta, and read/ edited the manuscript; MNB contributed to the design of the project, assisted in collecting seeds of Rhazya stricta, and read/edited the manuscript; MJS assisted in collecting seeds of Rhazya stricta and read/edited the manuscript; NAB contributed to the design of the project, assisted in collecting seeds of Rhazya stricta, and read/edited the manuscript; and RKJ contributed to the design of the project, assisted in collecting seeds of Rhazya stricta, and read/edited the manuscript. All authors read and approved the final manuscript.

\section{Acknowledgements}

The authors gratefully acknowledge the financial support from King Abdulaziz University Vice President for Educational Affairs Prof. Dr. Abdulrahman O. Alyoubi. We also thank Dhivya Arasappan for providing access to a draft assembly of the Rhazya stricta nuclear genome, the Genome Sequencing and Analysis Facility at the University of Texas at Austin for performing the Illumina sequencing, the Texas Advanced Computing Center at the University of Texas at Austin for access to supercomputers, and to Dhivya Arasappan, Chris Blazier, Erika Schwarz, Mao-Lun Weng, Jin Zhang and two anonymous reviewers for providing valuable comments on earlier versions of the manuscript.

Received: 5 March 2014 Accepted: 15 May 2014

Published: 28 May 2014

\section{References}

1. Ageel AM, Mossa JS, Al-Yahya MA, Tariq M, Al-Said MS: Plants Used in Saudi Folk Medicine. Riyadh, Saudia Arabia: King Saud University Press; 1987.

2. Ali BH, Bashir AK, Banna NR, Tanira MO: Central nervous system activity of Rhazya stricta Dence in mice. Clin Exp Pharmacol Physiol 1995, 22:248-253.

3. Ali BH, Bashir AK, Tanira MO: The effect of Rhazya stricta, a traditional medicinal plant, on the forced swimming test in rats. Pharmacol Biochem Behav 1998, 59:547-550.

4. Ali BH, Al-Qarawi AA, Bashir AK, Tanira MO: Phytochemistry, pharmacology and toxicity of Rhazya stricta Decne: A review. Phytother Res 2000, 14:229-234.

5. Gilani SA, Kikuchi A, Shinwari ZK, Khattak ZI, Watanabe KN: Phytochemical, pharmacological and ethnobotanical studies of Rhazya stricta Decne. Phytother Res 2007, 21:301-307.

6. Baeshen NA, Elkady Al, Abuzinadah OA, Mutwakil MH: Potential anticancer activity of the medicinal herb, Rhazya stricta, against human breast cancer. Afr J Biotechnol 2012, 11:8960-8972.

7. Graham IA, Besser K, Blumer S, Branigan CA, Czechowski T, Elias L, Guterman I, Harvey D, Isaac PG, Khan AM, Larson TR, Li Y, Pawson T, Penfield T, Rae AM, Rathbone DA, Reid S, Ross J, Smallwood MF, Segura V, Townsend T, Vyas D, Winzer T, Bowles D: The genetic map of Artemisia annua L. identifies loci affecting yield of the antimalarial drug artemisinin. Science 2010, 327:328-331. 
8. Li Y, Luo HM, Sun C, Song JY, Sun YZ, Wu Q, Wang N, Yao H, Steinmetz A, Chen SL: EST analysis reveals putative genes involved in glycyrrhizin biosynthesis. BMC Genomics 2010, 11:268

9. Fuller CW, Middendorf LR, Benner SA, Church GM, Harris T, Huang X, Jovanovich SB, Nelson JR, Schloss JA, Schwartz DC, Vezenov DV: The challenges of sequencing by synthesis. Nat Biotechnol 2009, 27:1013-1023.

10. Voelkerding KV, Dames SA, Durtschi JD: Next-generation sequencing: from basic research to diagnostics. Clin Chem 2009, 55:641-658

11. Metzker ML: Sequencing technologies - the next generation. Nat Rev Genet 2010, 11:31-46.

12. Treangen TJ, Salzberg SL: Repetitive DNA and next-generation sequencing: computational challenges and solutions. Nat Rev Genet 2012, 13:36-46.

13. Bock R: In Structure, function, and inheritance of plastid genomes, Cell and Molecular Biology of Plastids, Volume 19. Edited by Bock R. Berlin Heidelberg: Springer; 2007:29-63.

14. Jansen RK, Ruhlman TA: Plastid genomes of seed plants. In Genomics of Chloroplasts and Mitochondria. Edited by Bock R, Knoop V. New York: Springer; 2012:103-126

15. Terasawa K, Odahara M, Kabeya $Y$, Kikugawa $T$, Sekine $Y$, Fujiwara M, Sato N: The mitochondrial genome of the moss Physcomitrella patens sheds new light on mitochondrial evolution in land plants. Mol Biol Evol 2007, 24:699-709.

16. Sloan DB, Alverson AJ, Chuckalovcak JP, Wu M, McCauley DE, Palmer JD, Taylor DR: Rapid evolution of enormous, multichromosomal genomes in flowering plant mitochondria with exceptionally high mutation rates. PLOS Biol 2012, 10:e1001241.

17. Mower JP, Sloan DB, Alverson AJ: Plant mitochondrial genome diversity: the genomics revolution. In Plant genome diversity volume 1: plant genomes, their residents, and their evolutionary dynamics. Edited by Wendel JH. New York: Springer; 2012:123-144.

18. Ogihara Y, Yamazaki Y, Murai K, Kanno A, Terachi T, Shiina T, Miyashita N, Nasuda S, Nakamura C, Mori N, Takumi S, Murata M, Futo S, Tsunewaki K: Structural dynamics of cereal mitochondrial genomes as revealed by complete nucleotide sequencing of the wheat mitochondrial genome. Nucleic Acids Res 2005, 33:6235-6250.

19. Sugiyama $Y$, Watase $Y$, Nagase $M$, Makita $N$, Yagura S, Hirai A, Sugiura $M$ The complete nucleotide sequence and multipartite organization of the tobacco mitochondrial genome: comparative analysis of mitochondrial genomes in higher plants. Mol Genet Genomics 2005, 272:603-615.

20. Sloan DB, Alverson AJ, Storchová H, Palmer JD, Taylor DR: Extensive loss of translational genes in the structurally dynamic mitochondrial genome of the angiosperm Silene latifolia. BMC Evol Biol 2010, 10:274.

21. Alverson AJ, Zhuo S, Rice DW, Sloan DB, Palmer JD: The mitochondrial genome of the legume Vigna radiata and the analysis of recombination across short mitochondrial repeats. PLoS One 2011, 6:e16404.

22. Mower JP, Case AL, Floro ER, Willis JH: Evidence against equimolarity of large repeat arrangements and a predominant master circle structure of the mitochondrial genome from a monkeyflower (Mimulus guttatus) lineage with cryptic CMS. Genome Biol Evol 2012, 4:670-686.

23. Shedge V, Arrieta-Montiel M, Christensen AC, Mackenzie SA: Plant mitochondrial recombination surveillance requires unusual $\operatorname{Rec} A$ and MutS homologs. Plant Cell 2007, 19:1251-1264.

24. Hanson MR, Bentolila S: Interactions of mitochondrial and nuclear genes that affect male gametophyte development. Plant Cell 2004, 16:S154-S169.

25. Timmis JN, Ayliffe MA, Huang CY, Martin W: Endosymbiotic gene transfer: organelle genomes forge eukaryotic chromosomes. Nat Rev Genet 2004, 5:123-135.

26. Goremykin W, Salamini F, Velasco R, Viola R: Mitochondrial DNA of Vitis vinifera and the issue of rampant horizontal gene transfer. Mol Biol Evol 2009, 26:99-110.

27. Alverson AJ, Wei X, Rice DW, Stern DB, Barry K, Palmer JD: Insights into the evolution of mitochondrial genome size from complete sequences of Citrullus lanatus and Cucurbita pepo (Cucurbitaceae). Mol Biol Evol 2010, 27:1436-1448.

28. Rodríguez-Moreno L, González VM, Benjak A, Martí MC, Puigdomènech P, Aranda MA, Garcia-Mas J: Determination of the melon chloroplast and mitochondrial genome sequences reveals that the largest reported mitochondrial genome in plants contains a significant amount of DNA having a nuclear origin. BMC Genomics 2011, 12:424.

29. Rice DW, Alverson AJ, Richardson AO, Young GJ, Sanchez-Puerta MV, Munzinger J, Barry K, Boore JL, Zhang Y, de Pamphilis CW, Knox EB, Palmer JD:
Horizontal transfer of entire genomes via mitochondrial fusion in the angiosperm Amborella. Science 2013, 342:1468-1473.

30. Covello PS, Gray MW: RNA editing in plant mitochondria. Nature 1989, 341:662-666.

31. Steinhauser S, Beckert S, Capesius I, Malek O, Knoop V: Plant mitochondrial RNA editing. J Mol Evol 1999, 48:303-312.

32. Glaser E, Soll J: Targeting signals and import machinery of plastids and plant mitochondria. In Molecular Biology and Biotechnology of Plant Organelles: Chloroplasts and Mitochondria. Edited by Daniell H, Chase C. New York: Springer; 2004:385-418.

33. Adams KL, Palmer JD: Evolution of mitochondrial gene content: gene loss and transfer to the nucleus. Mol Phylogenet Evol 2003, 29:380-395.

34. Ueda M, Fujimoto M, Arimura S, Murata J, Tsutsumi N, Kadowaki K: Loss of the $r p / 32$ gene from the chloroplast genome and subsequent acquisition of a preexisting transit peptide within the nuclear gene in Populus. Gene 2007, 402:51-56.

35. Jansen RK, Saski C, Lee SB, Hansen AK, Daniell H: Complete plastid genome sequences of three rosids (Castanea, Prunus, Theobroma): evidence for at least two independent transfers of $r p / 22$ to the nucleus. Mol Biol Evol 2011, 28:835-847.

36. Liu SL, Zhuang Y, Zhang P, Adams KL: Comparative analysis of structural diversity and sequence evolution in plant mitochondrial genes transferred to the nucleus. Mol Biol Evol 2009, 26:875-891.

37. Raubeson LA, Jansen RK: Chloroplast genomes of plants. In Plant diversity and evolution: genotypic and phenotypic variation in higher plants. Edited by Henry RJ. Cambridge MA: CABl; 2005:45-68.

38. Weng ML, Blazier JC, Govindu M, Jansen RK: Reconstruction of the ancestral plastid genome in Geraniaceae reveals a correlation between genome rearrangements, repeats, and nucleotide substitution rates. Mol Biol Evol 2013, 31:645-659.

39. Straub SC, Cronn RC, Edwards C, Fishbein M, Liston A: Horizontal transfer of DNA from the mitochondrial to the plastid genome and its subsequent evolution in milkweeds (Apocynaceae). Genome Biol Evol 2013, 5:1872-1885.

40. Iorizzo M, Senalik D, Szklarczyk M, Grzebelus D, Spooner D, Simon P: De novo assembly of the carrot mitochondrial genome using next generation sequencing of whole genomic DNA provides first evidence of DNA transfer into an angiosperm plastid genome. BMC Plant Biol 2012, 12:61.

41. Conklin PL, Hanson MR: Recombination of plant mitochondrial genomes In Homologous Recombination and Gene Silencing in Plants. Edited by Paszkowski J. Netherlands: Springer; 1994:61-81.

42. Marienfeld J, Unseld M, Brandt P, Brennicke A: Genomic recombination of the mitochondrial atp6 gene in Arabidopsis thaliana at the protein processing site creates two different presequences. DNA Res 1996, 3:287-290.

43. Joyce PB, Gray MW: Chloroplast-like transfer RNA genes expressed in wheat mitochondria. Nucleic Acids Res 1989, 17:5461-5476.

44. Maréchal-Drouard L, Guillemaut P, Cosset A, Arbogast M, Weber F, Weil JH, Dietrich A: Transfer RNAs of potato (Solanum tubeosum) mitochondria have different genetic origins. Nucleic Acids Res 1990, 18:3689-3696.

45. Wang D, Wu YW, Shih AC, Wu CS, Wang YN, Chaw SM: Transfer of chloroplast genomic DNA to mitochondrial genome occurred at least 300 MYA. Mol Biol Evol 2007, 24:2040-2048.

46. Richardson AO, Rice DW, Young GJ, Alverson AJ, Palmer JD: The "fossilized" mitochondrial genome of Liriodendron tulipifera: ancestral gene content and order, ancestral editing sites, and extraordinarily low mutation rate. BMC Biol 2013, 11:29.

47. Kubo T, Nishizawa S, Sugawara A, Itchoda N, Estiati A, Mikami T: The complete nucleotide sequence of the mitochondrial genome of sugar beet (Beta vulgaris L.) reveals a novel gene for tRNA(Cys)(GCA). Nucleic Acids Res 2000, 28:2571-2576.

48. Holec S, Lange H, Kühn K, Alioua M, Börner T, Gagliardi D: Relaxed transcription in Arabidopsis mitochondria is counterbalanced by RNA stability control mediated by polyadenylation and polynucleotide phosphorylase. Mol Cell Biol 2006, 26:2869-2876.

49. Sanchez-Puerta MV, Cho Y, Mower JP, Alverson AJ, Palmer JD: Frequent, phylogenetically local horizontal transfer of the cox 1 group I Intron in flowering plant mitochondria. Mol Biol Evol 2008, 25:1762-1777.

50. Cusimano N, Zhang LB, Renner SS: Reevaluation of the cox 1 group I intron in Araceae and angiosperms indicates a history dominated by loss rather than horizontal transfer. Mol Biol Evol 2008, 25:265-276.

51. Mower JP, Jain K, Hepburn NJ: The role of horizontal transfer in shaping the plant mitochondrial genome. In Advances in Botanical Research Volume 
63: Mitochondrial Genome Evolution. Edited by Maréchal-Drouard L. New York: Academic Press; 2012:41-69.

52. Adams KL, Qiu YL, Stoutemyer M, Palmer JD: Punctuated evolution of mitochondrial gene content: High and variable rates of mitochondrial gene loss and transfer to the nucleus during angiosperm evolution. Proc Natl Acad Sci U S A 2002, 99:9905-9912.

53. Adams KL, Rosenblueth M, Qiu YL, Palmer JD: Multiple losses and transfers to the nucleus of two mitochondrial succinate dehydrogenase genes during angiosperm evolution. Genetics 2001, 158:1289-1300.

54. Ong HC, Palmer JD: Pervasive survival of expressed mitochondrial rps14 pseudogenes in grasses and their relatives for 80 million years following three functional transfers to the nucleus. BMC Evol Biol 2006, 6:55.

55. Adams KL, Song K, Roessler PG, Nugent JM, Doyle JL, Doyle JJ, Palmer JD: Intracellular gene transfer in action: dual transcription and multiple silencings of nuclear and mitochondrial cox2 genes in legumes. Proc Natl Acad Sci U S A 1999, 96:13863-13868

56. Sandoval P, León G, Gómez I, Carmona R, Figueroa P, Holuigue L, Araya A, Jordana X: Transfer of RPS14 and RPL5 from the mitochondrion to the nucleus in grasses. Gene 2004, 324:139-147.

57. Choi C, Liu Z, Adams KL: Evolutionary transfers of mitochondrial genes to the nucleus in the Populus lineage and coexpression of nuclear and mitochondrial Sdh4 genes. New Phytol 2006, 172:429-439.

58. Maréchal A, Brisson N: Recombination and the maintenance of plant organelle genome stability. New Phytol 2010, 186:299-317.

59. Doyle JJ, Doyle JL: A rapid DNA isolation procedure for small quantities of fresh leaf tissue. Phytochem Bull 1987, 19:11-15.

60. Koren S, Schatz MC, Walenz BP, Martin J, Howard JT, Ganapathy G, Wang Z, Rasko DA, McCombie WR, Jarvis ED, Phillippy AM: Hybrid error correction and de novo assembly of single-molecule sequencing reads. Nat Biotechnol 2012, 30:693-700.

61. Zerbino DR, Birney E: Velvet: algorithms for de novo short read assembly using de Bruijn graphs. Genome Res 2008, 18:821-829.

62. Biomatters: Geneious R6 v.6.1.6. [http://www.geneious.com/]

63. Wyman SK, Jansen RK, Boore JL: Automatic annotation of organellar genomes with DOGMA. Bioinformatics 2004, 20:3252-3255

64. Dombrovska O, Qiu YL: Distribution of introns in the mitochondrial gene nad1 in land plants: phylogenetic and molecular evolutionary implications. Mol Phylogenet Evol 2004, 32:246-263.

65. Lowe TM, Eddy SR: tRNAscan-SE: A program for improved detection of transfer RNA genes in genomic sequence. Nucleic Acids Res 1997, 25:955-964.

66. Lohse M, Drechsel O, Kahlau S, Bock R: OrganellarGenomeDRAW - a suite of tools for generating physical maps of plastid and mitochondrial genomes and visualizing expression data sets. Nucleic Acids Res 2013, 41:W575-W581.

67. Krzywinski M, Schein J, Birol I, Connors J, Gascoyne R, Horsman D, Jones SJ, Marra MA: Circos: an information aesthetic for comparative genomics. Genome Res 2009, 19:1639-1645.

68. Untergasser A, Cutcutache I, Koressaar T, Ye J, Faircloth BC, Remm M Rozen SG: Primer3 - new capabilities and interfaces. Nucleic Acids Res 2012, 40:e115.

69. The NCBI ORF finder. [http://www.ncbi.nlm.nih.gov/gorf/gorf.html]

70. Krogh A, Larsson B, von Heijne G, Sonnhammer EL: Predicting transmembrane protein topology with a hidden Markov model: application to complete genomes. J Mol Biol 2001, 305:567-580.

71. Kohany O, Gentles AJ, Hankus L, Jurka J: Annotation, submission and screening of repetitive elements in Repbase: RepbaseSubmitter and Censor. BMC Bioinformatics 2006, 7:474.

72. Mower JP: The PREP Suite: Predictive RNA editors for plant mitochondrial genes, chloroplast genes and user-defined alignments. Nucleic Acids Res 2009, 37:W253-W259.

73. Lenz H, Knoop V: PREPACT 2.0: Predicting C-to-U and U-to-C RNA editing in organelle genome sequences with multiple references and curated RNA editing annotation. Bioinfrom Biol Insights 2013, 7:1-19.

74. Zhang J, Ruhlman TA, Mower JP, Jansen RK: Comparative analyses of two Geraniaceae transcriptomes using next-generation sequencing BMC Plant Biol 2013, 13:228.

75. Grabherr MG, Haas BJ, Yassour M, Levin JZ, Thompson DA, Amit I, Adiconis X, Fan L, Raychowdhury R, Zeng Q, Chen Z, Mauceli E, Hacohen N, Gnirke A Rhind N, di Palma F, Birren BW, Nusbaum C, Lindblad-Toh K, Friedman N,
Regev A: Full-length transcriptome assembly from RNA-Seq data without a reference genome. Nat Biotechnol 2011, 29:644-652.

76. Emanuelsson O, Brunak S, von Heijne G, Nielsen H: Locating proteins in the cell using TargetP, SignalP, and related tools. Nat Protoc 2007, 2:953-971.

77. Small I, Peeters N, Legeai F, Lurin C: Predotar: A tool for rapidly screening proteomes for N-terminal targeting sequences. Proteomics 2004, 4:1581-1590.

78. Goodstein DM, Shu S, Howson R, Neupane R, Hayes RD, Fazo J, Mitros T, Dirks W, Hellsten U, Putnam N, Rokhsar DS: Phytozome: a comparative platform for green plant genomics. Nucleic Acids Res 2012, 40:D1178-D1186.

79. Marchler-Bauer A, Zheng C, Chitsaz F, Derbyshire MK, Geer LY, Geer RC, Gonzales NR, Gwadz M, Hurwitz DI, Lanczycki CJ, Lu F, Lu S, Marchler GH, Song JS, Thanki N, Yamashita RA, Zhang D, Bryant SH: CDD: conserved domains and protein three-dimensional structure. Nucleic Acids Res 2013, 41:D348-D352.

80. Edgar RC: MUSCLE: multiple sequence alignment with high accuracy and high throughput. Nucleic Acids Res 2004, 32:1792-1797.

81. Stamatakis A: RAxML-VI-HPC: maximum likelihood-based phylogenetic analyses with thousands of taxa and mixed models. Bioinformatics 2006, 22:2688-2690

82. Geer LY, Domrachev M, Lipman DJ, Bryant SH: CDART: protein homology by domain architecture. Genome Res 2002, 12:1619-1623.

\section{doi:10.1186/1471-2164-15-405}

Cite this article as: Park et al:: Complete sequences of organelle genomes from the medicinal plant Rhazya stricta (Apocynaceae) and contrasting patterns of mitochondrial genome evolution across asterids. BMC Genomics 2014 15:405.

\section{Submit your next manuscript to BioMed Central and take full advantage of:}

- Convenient online submission

- Thorough peer review

- No space constraints or color figure charges

- Immediate publication on acceptance

- Inclusion in PubMed, CAS, Scopus and Google Scholar

- Research which is freely available for redistribution 